\title{
Light Combining for Interferometric Switching
}

\author{
Marco Masi, ${ }^{1}$ Mattia Mancinelli, ${ }^{2}$ Paolo Bettotti, ${ }^{2}$ and Lorenzo Pavesi ${ }^{2}$ \\ ${ }^{1}$ Institut des Nanotechnologies de Lyon, Université de Lyon, INL-UMR5270, CNRS, INSA de Lyon, 69621 Villeurbanne, France \\ ${ }^{2}$ Nanoscience Laboratory, Department of Physics, University of Trento, Via Sommarive 14, 38121 Trento, Italy
}

Correspondence should be addressed to Marco Masi, marco.masi@gmail.com

Received 4 February 2012; Accepted 11 April 2012

Academic Editor: Francesco Prudenzano

Copyright ( 2012 Marco Masi et al. This is an open access article distributed under the Creative Commons Attribution License, which permits unrestricted use, distribution, and reproduction in any medium, provided the original work is properly cited.

\begin{abstract}
Interferometric switching as a routing method in sequence of coupled optical microresonators is explored. Mach-Zhender interferometry is extended to systems of side-coupled integrated sequences of resonators (SCISSORs) and coupled resonators optical waveguides (CROWs). We generalized Coupled Mode Theory (CMT) to a system of three coupled waveguides. The two bus interferometric switching functions of SCISSOR and CROW resonant structures are investigated. A novel switching device based on three input phase modulation ports is presented. This device displays a wide range of switching behaviors which might lead to new interesting applications.
\end{abstract}

\section{Introduction}

The tremendous growth of communication services and information technologies demands new and enhanced networking capabilities. Switching is one of the main functions of communication systems and networks. In particular, novel optical switching technologies have the potential to play a decisive role in future telecommunication and information processing systems. The extensive deployment of wavelength division multiplexing (WDM) technologies demands for high-speed, scalable, and rapidly reconfigurable network switching. Several optical switching technologies exist, like electrooptic (EO), acoustooptic (AO), thermooptic (TO), optomechanical (OM), and optical amplifier (OA) based switching. Refractive index modulation, inducing phase differences for switching functionalities, resorts especially to EO or TO effects. These are widely used in directional couplers, Mach-Zehnder interferometers, and multi-mode interference (MMI) switches (for a good review see, e.g., [1]). The present paper focuses on some possible extensions of these interferometric techniques by means of sequences of microoptical resonator systems, like SCISSORs (sidecoupled integrated spaced-sequences of resonators) and CROWs (coupled resonator optical waveguides) [2-6]. A particular combination of these allows to use three input waveguides for amplitude and phase modulation. The advantage of a system of resonators over conventional singleresonator scheme coupling is its larger spectral band, the possibility to operate through interferometry on several channels and its higher design flexibility. In particular, this latter aspect allows for a diversity of behaviors compared to usual directional coupling switching.

The practical realization of such kind of phase switching devices is, however, still limited by present photonic fabrication tolerances. Phase shifting of light requires the same interferometric precision as the device operation itself. For example, the phase difference between two interfering light beams needs to be tightly controlled at the subwavelength level to provide efficient switching. Even more sensitive to small deviations from the nominal parameters are devices where coherent addition of signals is achieved by arranging complex systems of resonator chains. These require nanometer level accuracy in the fabrication of the single resonators and their mutual spacings. For instance, coupled resonator induced transparency (CRIT) effects emerge easily only for few nanometer deviations [7-12]. However, while present optical lithography still suffers of few nanometers imprecision needing further improvements, the next generation optical lithography is likely to achieve an order of magnitude leap in accuracy paving the way to novel interferometric devices [13]. Also, the fabrication of electrooptic siliconphotonic modulators which are capable of providing smooth 
and uniform phase shifts over a broad spectrum is a topic of intense research and promises to find a wide area of applications [14]. Elsewhere we have demonstrated the practical realization of a novel silicon photonic interferometric switching device showing that resonator phase switching is indeed feasible [12]. Therefore, it is interesting to explore from the theoretical point of view some extensions of conventional directional coupling methods which could have potential applications in upcoming interferometric devices.

The next section will briefly summarize the basics of coupled mode theory (CMT) which will be particularly useful in our context. Section 2 will set the stage for interferometric switching between three waveguides which in turn will be used in Section 3 where interferometric switching with a dual bus waveguide resonator system is described. Section 4 will extend this to a particular arrangement of three-bus resonator systems.

\section{CMT and Phase Switching for the Double-Sided Symmetric Codirectional Coupler}

CMT has grown to a vast subject in the last three decades (for a good introduction see e.g., [15]). Let us quickly recall some standard equations which describe in particular the coupling between two waveguides.

We assume that the two waveguides have the same geometry and propagation constants $\beta$, and they are homogenous and isotropic. Material or other types of losses are negligible. Within these assumptions, the fields in the first and second waveguide are given by the solution of the following coupled differential equations:

$$
\begin{aligned}
& \frac{\partial A_{1}(z)}{\partial z}=i c A_{2}(z), \\
& \frac{\partial A_{2}(z)}{\partial z}=i c A_{1}(z),
\end{aligned}
$$

with $A_{1}(z)$ and $A_{2}(z)$ are the $z$ dependent parts of the fields, and $c$ a mutual mode coupling coefficient. $c$ is obtained from perturbation theory by the amplitudes cross-sectional integral over the section of the two waveguides of the codirectional coupler and has the dimension of an inverse length. If we impose as initial conditions

$$
A_{1}(0)=A_{1} e^{i \phi_{1}}, \quad A_{2}(0)=A_{2} e^{i \phi_{2}},
$$

then the solutions at $z$ are

$$
\begin{aligned}
& A_{1}(z)=t A_{1} e^{i \phi_{1}}+i \kappa A_{2} e^{i \phi_{2}}, \\
& A_{2}(z)=i \kappa A_{1} e^{i \phi_{1}}+t A_{2} e^{i \phi_{2}},
\end{aligned}
$$

with

$$
\kappa=\sin (c z) e^{i \beta z}, \quad t=\cos (c z) e^{i \beta z},
$$

being $\kappa$ and $t$ the cross- and through-coupling coefficients, respectively, and $e^{i \beta z}$ accounts for the phase shift. $\kappa$ and $t$ are given by

$$
\kappa=\sin \left(\frac{\pi L}{2 L_{c}}\right) e^{i \beta L}, \quad t=\cos \left(\frac{\pi L}{2 L_{c}}\right) e^{i \beta L},
$$

where

$$
L_{c}=\frac{\lambda}{2\left(n_{\text {effe }}(\lambda)-n_{\text {effo }}(\lambda)\right)}
$$

is the coupling length, $\lambda$ the wavelength, $L$ the length of the coupling section, while $n_{\text {effe }}$ and $n_{\text {effo }}$ are the even and odd mode wavelength-dependent effective indexes, respectively. Then, from (4) and (5), one obtains

$$
c=\frac{\pi}{2 L_{c}} .
$$

From (3), it follows that

$$
\begin{aligned}
& \left|A_{1}(z)\right|^{2}=|t|^{2}\left|A_{1}\right|^{2}+|\kappa|^{2}\left|A_{2}\right|^{2}+2|\kappa||t|\left|A_{1}\right|\left|A_{2}\right| \sin \Delta \phi, \\
& \left|A_{2}(z)\right|^{2}=|\kappa|^{2}\left|A_{1}\right|^{2}+|t|^{2}\left|A_{2}\right|^{2}-2|\kappa||t|\left|A_{1}\right|\left|A_{2}\right| \sin \Delta \phi,
\end{aligned}
$$

with $\Delta \phi=\phi_{1}-\phi_{2}$. For simplicity in (8), the dependence of the coupling coefficients on $z$ is omitted. Power conservation condition for the lossless system follows straightforwardly from (5), or (8), as $|t|^{2}+|\kappa|^{2}=1$.

If we set $A_{2}=0$, we obtain the well-known power exchange expressions for the single-sided codirectional coupler made of two equal waveguides

$$
\begin{aligned}
& \left|A_{1}(z)\right|^{2}=\cos ^{2}(c z)\left|A_{1}\right|^{2}=|t|^{2}\left|A_{1}\right|^{2}, \\
& \left|A_{2}(z)\right|^{2}=\sin ^{2}(c z)\left|A_{1}\right|^{2}=|\kappa|^{2}\left|A_{1}\right|^{2} .
\end{aligned}
$$

The Mach-Zehnder modulator is obtained when two signals with same amplitude $\left(A_{1}=A_{2}=A\right)$ are coupled into the waveguides. In fact, (8) becomes

$$
\begin{aligned}
& \left|A_{1}(z)\right|^{2}=|A|^{2}(1+2|\kappa||t| \sin \Delta \phi), \\
& \left|A_{2}(z)\right|^{2}=|A|^{2}(1-2|\kappa||t| \sin \Delta \phi),
\end{aligned}
$$

which shows that, when $\Delta \phi=0$, no power exchange between the two waveguides occurs, while, for $\Delta \phi=m(\pi / 2)(m$ an integer), the power oscillates between the two waveguides depending on the coupling section length, $L$. If $c L=\pi / 4$ and $\Delta \phi= \pm \pi / 2$, then all the power is transferred into one or the other waveguide.

Let us now extend (1) to the situation of Figure 1, where three waveguides couple. We call the central waveguide the Drop waveguide for a reason that will be clear in the following. We consider the case of a Drop signal $A_{D}$ excited by the fields $A_{1}$ and $A_{2}$ in the upper and lower waveguides. Then, neglecting the direct coupling between the two outer waveguides, we write a set of three coupled differential equations:

$$
\begin{gathered}
\frac{\partial A_{1}(z)}{\partial z}=i c A_{D}(z), \\
\frac{\partial A_{D}(z)}{\partial z}=i c A_{1}(z)+i c A_{2}(z), \\
\frac{\partial A_{2}(z)}{\partial z}=i c A_{D}(z) .
\end{gathered}
$$

We impose the initial conditions

$$
\begin{aligned}
A_{1}(0) & =A_{1} e^{i \phi_{1}}, \\
A_{D}(0) & =A_{D} e^{i \phi_{D}}, \\
A_{2}(0) & =A_{2} e^{i \phi_{2}},
\end{aligned}
$$




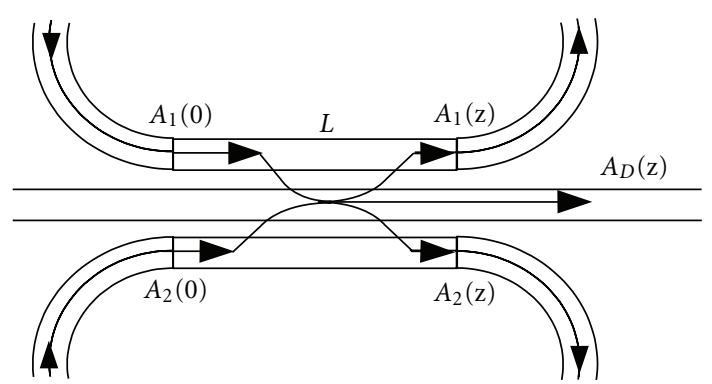

Figure 1: Double sided symmetric codirectional coupler.

where $\phi_{1}, \phi_{1}$, and $\phi_{D}$ express the phase of $A_{1}, A_{2}$, and $A_{D}$, respectively. The solutions of (11) are

$$
\begin{aligned}
& A_{1}(z)=t^{\prime} A_{1} e^{i \phi_{1}}+i \kappa_{D} A_{D} e^{i \phi_{D}}-\kappa^{\prime} A_{2} e^{i \phi_{2}}, \\
& A_{D}(z)=i \kappa_{D} A_{1} e^{i \phi_{1}}+t_{D} A_{D} e^{i \phi_{D}}+i \kappa_{D} A_{2} e^{i \phi_{2}}, \\
& A_{2}(z)=-\kappa^{\prime} A_{1} e^{i \phi_{1}}+i \kappa_{D} A_{D} e^{i \phi_{D}}+t^{\prime} A_{2} e^{i \phi_{2}},
\end{aligned}
$$

with

$$
\begin{aligned}
t^{\prime} & =\cos ^{2}\left(\frac{c z}{\sqrt{2}}\right) e^{i \beta z}, & t_{D} & =\cos (\sqrt{2} c z) e^{i \beta z}, \\
\kappa^{\prime} & =\sin ^{2}\left(\frac{c z}{\sqrt{2}}\right) e^{i \beta z}, & \kappa_{D} & =\frac{1}{\sqrt{2}} \sin (\sqrt{2} c z) e^{i \beta z} .
\end{aligned}
$$

The term $1 / \sqrt{2}$ in (15) is a consequence of the symmetry of the system (symmetric coupling and geometry). For simplicity, let us consider $A_{D}=0$, then

$$
\begin{aligned}
\left|A_{1}(z)\right|^{2}= & \left|t^{\prime}\right|^{2}\left|A_{1}\right|^{2}+\left|\kappa^{\prime}\right|^{2}\left|A_{2}\right|^{2} \\
& -2\left|\kappa^{\prime}\right|\left|t^{\prime}\right|\left|A_{1}\right|\left|A_{2}\right| \cos \Delta \phi, \\
\left|A_{D}(z)\right|^{2}= & \left|\kappa_{D}\right|^{2}\left(\left|A_{1}\right|^{2}+\left|A_{2}\right|^{2}+2\left|A_{1}\right|\left|A_{2}\right| \cos \Delta \phi\right), \\
\left|A_{2}(z)\right|^{2}= & \left|\kappa^{\prime}\right|^{2}\left|A_{1}\right|^{2}+\left|t^{\prime}\right|^{2}\left|A_{2}\right|^{2} \\
& -2\left|\kappa^{\prime}\right|\left|t^{\prime}\right|\left|A_{1}\right|\left|A_{2}\right| \cos \Delta \phi .
\end{aligned}
$$

If the system is lossless, power conservation implies $\left|k^{\prime}\right|^{2}+\left|k_{D}\right|^{2}+\left|t^{\prime}\right|^{2}=1$. Despite not being directly coupled to each others, (16) and (18) show a possible crosstalk between the outer waveguides through the intermediate Drop port (crossing of arrows through the Drop port shown in Figure 1). This can be quantified by using as initial condition, for example, $A_{2}=0$ which implies from (16)-(18)

$$
\begin{aligned}
\left|A_{1}(z)\right|^{2} & =\left|A_{1}\right|^{2} \cos ^{4}\left(\frac{c z}{\sqrt{2}}\right), \\
\left|A_{D}(z)\right|^{2} & =\frac{\left|A_{1}\right|^{2}}{2} \sin ^{2}(\sqrt{2} c z), \\
\left|A_{2}(z)\right|^{2} & =\left|A_{1}\right|^{2} \sin ^{4}\left(\frac{c z}{\sqrt{2}}\right) .
\end{aligned}
$$

Equation (19) is plotted in Figure 2 in normalized intensity and distance. These show that the signal from the upper (lower) waveguide is never completely recovered in the Drop port because part of it recouples towards the lower (upper)

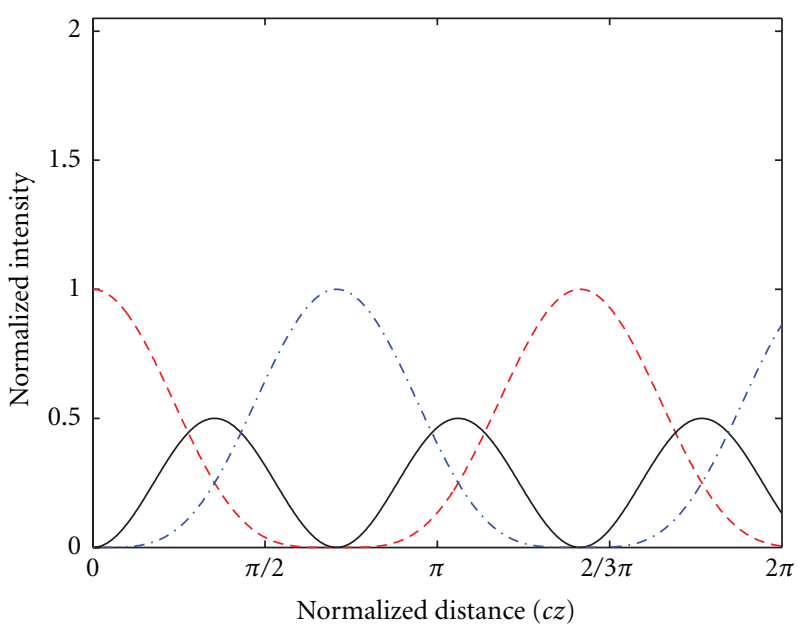

Figure 2: Power transfer in the waveguides of the ds-coupler for one input signal only ((16), (17), and (18), with initial conditions $\left.A_{1}=1, A_{2}=0\right)$. Red dashed line: amplitude $A_{1}(c z)$; blue dotteddashed line: amplitude $A_{2}(c z)$; black solid line: amplitude $A_{D}(c z)$.

waveguide. The Drop behaves like a "power transiting" port, and the amount of light that crosses the structure can be considered as a measure of crosstalk. However, we will see that this interpretation must be taken with caution.

In fact, if we consider a same input signal $\left(A_{1}=A_{2}=A\right)$ on the top and bottom waveguides, (16)-(18) simplify to

$$
\begin{gathered}
\left|A_{1}(z)\right|^{2}=\left|A_{2}(z)\right|^{2}=|A|^{2}\left(1-\frac{1}{2} \sin ^{2}(\sqrt{2} c z)(1+\cos \Delta \phi)\right), \\
\left|A_{D}(z)\right|^{2}=|A|^{2}(1+\cos \Delta \phi) \sin ^{2}(\sqrt{2} c z) .
\end{gathered}
$$

The case for in phase signals $(\Delta \phi=0)$ is shown in Figure 3(a). In this case, the energy transfer between the Drop and external ports occurs harmonically. A 100\% power transfer to the Drop port occurs when

$$
L=\frac{\pi}{2 \sqrt{2} c}=\frac{L_{c}}{\sqrt{2}}
$$

Figures 3(b) and 3(c) show this case for a phase difference of $\Delta \phi=\pi / 4$ and $\Delta \phi=(3 / 4) \pi$ : the power in the outer waveguides 1 and 2 (magenta dotted-dashed line) oscillates around the initial input value but is never zero, while the Drop signal (black solid line) oscillates according to the propagation length like in the previous case, but with less maximum intensity. If instead a phase difference of $\Delta \phi=\pi$ is applied (Figure 3(d)), there is no power in the Drop port.

Therefore, a particularly interesting situation occurs when a ds-coupler has a coupling section long as in (21) $(c z=(1 / \sqrt{2})(\pi / 2)$ in Figure 3). In fact, for two equal input signals the ds-coupler acts as an interferometric switch: for $\Delta \phi=0$, all the power goes into the drop port (Figure 4), while, for $\Delta \phi=\pi$, all the power stays in waveguides 1 and 2 (Figure 5). Under these circumstances, the crosstalk between waveguide 1 and 2 has been suppressed. At first, this seems to be at odds with what shown in Figure 2 where a 


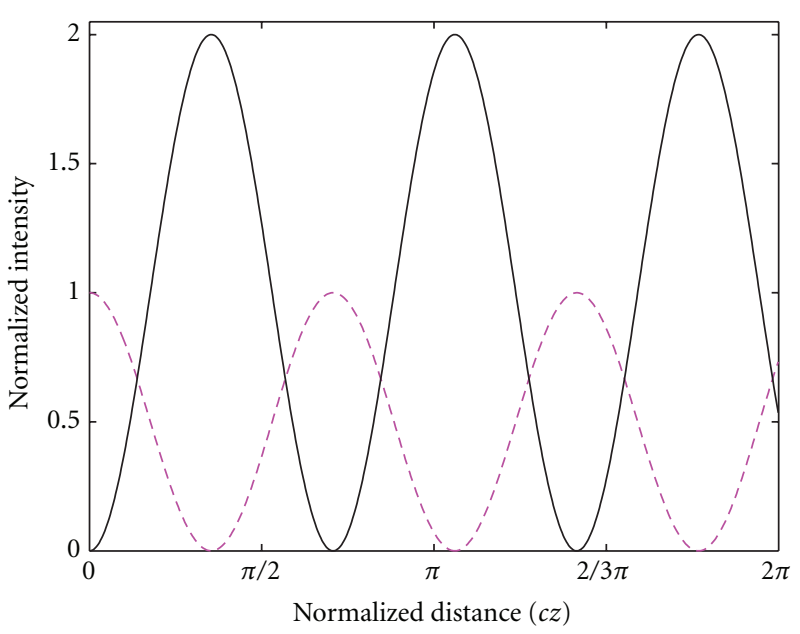

(a)

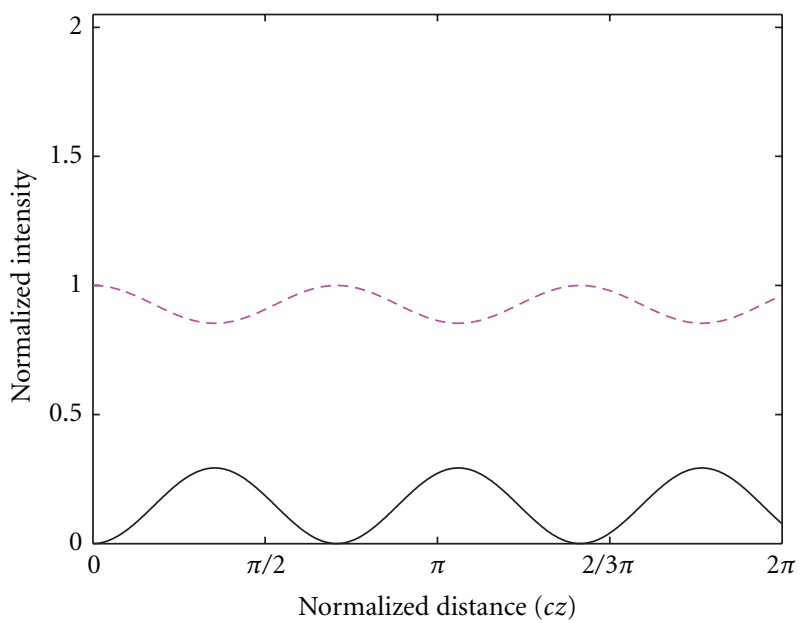

(c)

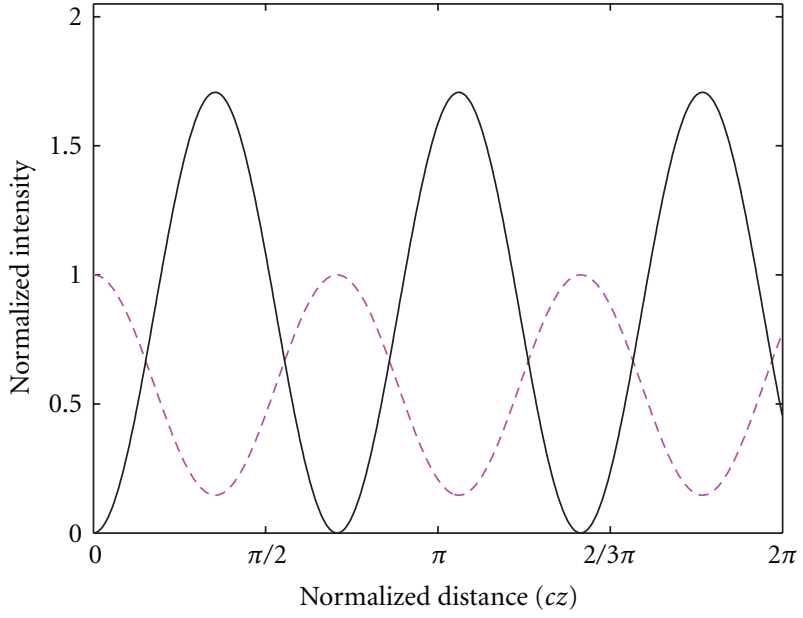

(b)

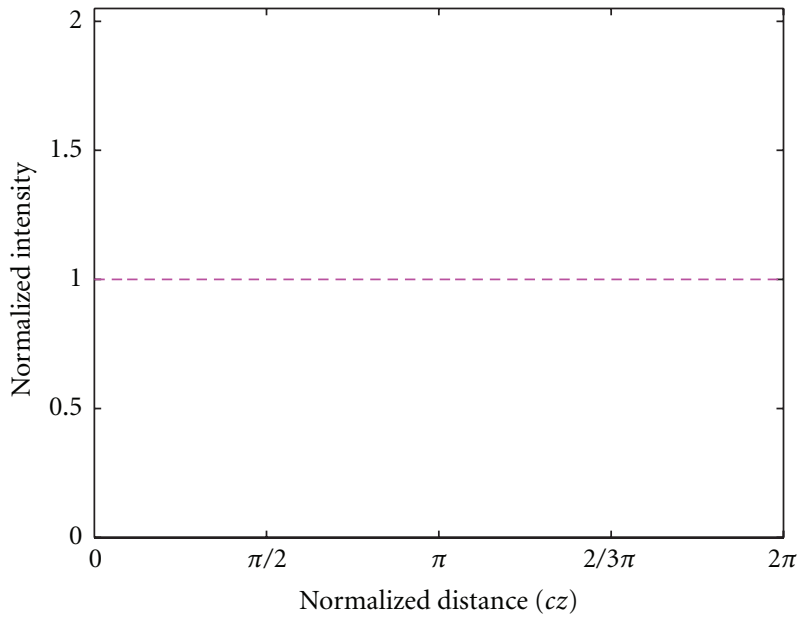

(d)

Figure 3: Power transfer in the waveguides of the ds-coupler for two equal input signals ((16), (17), and (18), with initial conditions $A_{1}=1$, $A_{2}=1, \Delta \phi=(0, \pi / 4,(3 / 4) \pi, \pi)$ in (a), (b), (c), (d), resp.). Magenta dotted-dashed line: amplitude $A_{1}(c z)$ and $A_{2}(c z)$; black solid line: amplitude $A_{D}(c z)$.

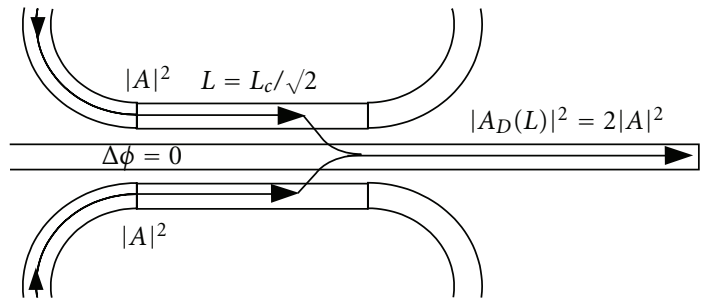

FIGURE 4: The ds-coupler switch in phase: all the power goes into the Drop port.

significant power transfer from waveguide 1 to waveguide 2 is observed. This apparent contradiction is explained by the fact that the signal in waveguide 1 (waveguide 2) undergoes twice a phase change of $\pi / 2$ (evanescent waves have always a $\pi / 2$ phase difference with respect to the waveguide core field phase) and the net $\pi$ dephased cross coupled wave interferes

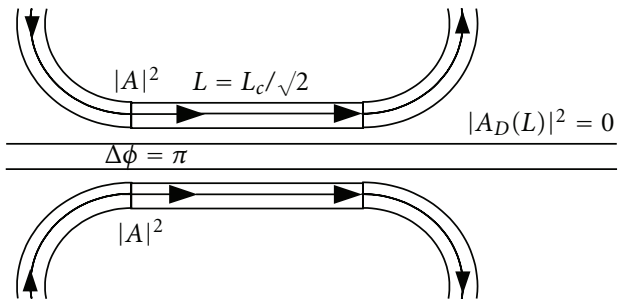

Figure 5: The ds-coupler switch out of phase: no power couples into the Drop port.

destructively in waveguide 2 (waveguide 1). This interference effect leads to a mutual subtraction of the cross-coupled powers.

For completeness, we mention also the case when the light is injected only in the Drop waveguide, that is,

$$
A_{1}(0)=0, \quad A_{D}(0)=A_{D}, \quad A_{2}(0)=0,
$$




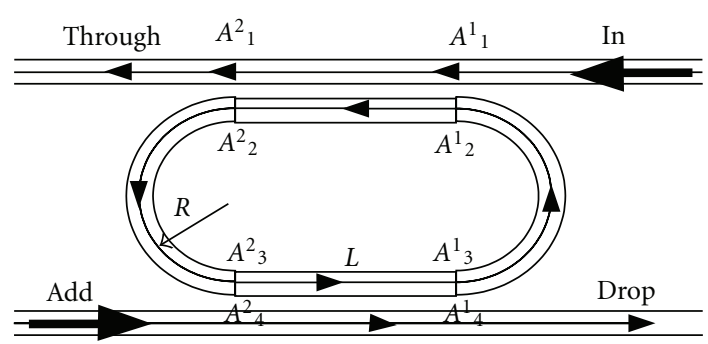

FIGURE 6: The single racetrack switch.

then

$$
\begin{gathered}
A_{1}(z)=A_{2}(z)=\frac{i A_{D} \sin (\sqrt{2} c z)}{\sqrt{2}} e^{i \beta z}, \\
A_{D}(z)=A_{D} \cos (\sqrt{2} c z) e^{i \beta z},
\end{gathered}
$$

whereby of course

$$
\begin{gathered}
\left|A_{1}(z)\right|^{2}=\left|A_{2}(z)\right|^{2}=\frac{\left|A_{D}\right|^{2}}{2} \sin ^{2}(\sqrt{2} c z)=\left|A_{D}\right|^{2} \frac{\left|\kappa_{D}\right|^{2}}{2}, \\
\left|A_{D}(z)\right|^{2}=\left|A_{D}\right|^{2} \cos ^{2}(\sqrt{2} c z)=\left|A_{D}\right|^{2}\left|t_{D}\right|^{2} .
\end{gathered}
$$

These show that the power is equally distributed among the outer waveguides. Note that by imposing a coupling section length equal to the value given by (21), it leads to a complete power transfer into waveguide 1 and 2, that is, the ds-coupler can be used as a splitter. Its advantage over other splitting devices, as Y-branches or MMI splitters, is that it is less sensitive to imbalances, provided that the spacing difference between the waveguides arising due to fabrication imperfections is negligible. Its disadvantage is obviously that it is wavelength dependent.

\section{Dual Bus Resonator Interferometric Switch}

A further extension of the formalism is to consider a single resonator as in Figure 6. We consider a racetrack resonator so that we can apply the CMT extension developed in Section 2. For simplicity, the bus-waveguide gaps and the coupling section lengths are the same everywhere. To apply the transfer matrix approach [16-21], a first step is to relate the amplitudes $\left(A_{1}^{1}, A_{2}^{1}\right)$ with $\left(A_{1}^{2}, A_{2}^{2}\right)$ (see Figure 6 for the definitions). From (3), we have:

$$
\left(\begin{array}{l}
A_{1}^{2} \\
A_{2}^{2}
\end{array}\right)=\mathbf{M}\left(\begin{array}{l}
A_{1}^{1} \\
A_{2}^{1}
\end{array}\right)=\left(\begin{array}{cc}
t & i \kappa \\
i \kappa & t
\end{array}\right)\left(\begin{array}{l}
A_{1}^{1} \\
A_{2}^{1}
\end{array}\right)
$$

In order to "transfer" the signal, it is more appropriate to write the relationships $\left(A_{1}^{1}, A_{1}^{2}\right) \rightarrow\left(A_{2}^{1}, A_{2}^{2}\right)$. For which one obtains the coupling matrix $\mathrm{K}$ :

$$
\left(\begin{array}{l}
A_{2}^{1} \\
A_{2}^{2}
\end{array}\right)=\mathbf{K}\left(\begin{array}{l}
A_{1}^{1} \\
A_{1}^{2}
\end{array}\right)=\frac{1}{i|k|}\left(\begin{array}{cc}
-|t| & e^{-i \beta L} \\
-e^{i \beta L} & |t|
\end{array}\right)\left(\begin{array}{l}
A_{1}^{1} \\
A_{1}^{2}
\end{array}\right),
$$

where, for the straight sections, the lossless case $\left(\left|\kappa_{1}\right|^{2}+\right.$ $\left|t_{1}\right|^{2}=1$ ) was considered. We instead assume bending losses

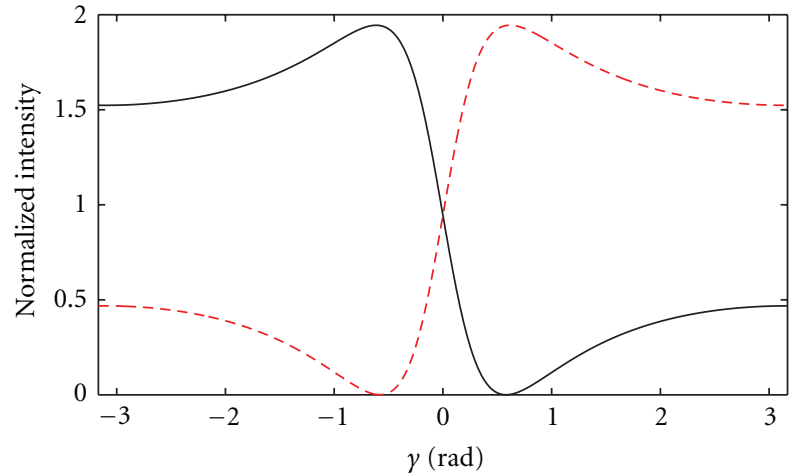

Figure 7: The dual-bus single resonator Fano resonance spectrum for $\Delta \phi=\pi / 2$ (Drop: black solid line, Through: red dashed line).

in the racetrack. The (forward and backward) internal propagation matrix $\mathbf{P}$ is

$$
\left(\begin{array}{l}
A_{3}^{2} \\
A_{3}^{1}
\end{array}\right)=\mathbf{P}\left(\begin{array}{l}
A_{2}^{1} \\
A_{2}^{2}
\end{array}\right)=\left(\begin{array}{cc}
0 & a e^{i \beta \pi R} \\
\frac{1}{a} e^{-i \beta \pi R} & 0
\end{array}\right)\left(\begin{array}{l}
A_{2}^{1} \\
A_{2}^{2}
\end{array}\right),
$$

where $a=e^{-\alpha \pi R}$ is the half round trip loss factor, $\alpha$ the total loss per unit length, and $R$ the curvature radius.

Equations (26) and (27) define a transfer matrix $\mathrm{T}^{\mathrm{PK}}$ as

$$
\left(\begin{array}{l}
A_{3}^{2} \\
A_{3}^{1}
\end{array}\right)=\mathrm{T}^{\mathrm{PK}}\left(\begin{array}{l}
A_{1}^{1} \\
A_{1}^{2}
\end{array}\right)=\mathbf{P K}\left(\begin{array}{l}
A_{1}^{1} \\
A_{1}^{2}
\end{array}\right) .
$$

With the definition of the Input, Through, Drop, and Add ports as in Figure 6, we define $A_{\mathrm{In}}, A_{\mathrm{Th}}, A_{D}$, and $A_{\mathrm{Ad}}$ the respective field amplitudes. Therefore,

$$
\left(\begin{array}{c}
A_{\mathrm{Ad}} \\
A_{D}
\end{array}\right)=\mathrm{T}^{\mathrm{l}}\left(\begin{array}{c}
A_{\mathrm{In}} \\
A_{\mathrm{Th}}
\end{array}\right)=\mathbf{K T}^{\mathrm{PK}}\left(\begin{array}{c}
A_{\text {In }} \\
A_{\mathrm{Th}}
\end{array}\right) .
$$

By rearranging the elements of the transfer matrix $\mathbf{T}^{\mathbf{1}}$ into the scattering matrix $\mathbf{S}^{1}$, one obtains

$$
\left(\begin{array}{c}
A_{\mathrm{Th}} \\
A_{D}
\end{array}\right)=S^{1}\left(\begin{array}{c}
A_{\mathrm{In}} \\
A_{\mathrm{Ad}}
\end{array}\right) .
$$

To simulate the device of Figure 6, we use the following parameters: bent curvature radius of $3.25 \mu \mathrm{m}$, gap spacing of $0.2 \mu \mathrm{m}$, silicon waveguides width of $0.45 \mu \mathrm{m}$, silicon waveguide core height of $0.22 \mu \mathrm{m}, \mathrm{SiO}_{2}$ cladding of $0.75 \mu \mathrm{m}$, and $-0.03 \mathrm{~dB} / 90^{\circ}$ bent loss in TE polarization [22].

The first interesting situation is when we inject two input signals, one in the Input port and the other in the Add port such that $A_{\mathrm{In}}=e^{i \phi_{1}}$ and $A_{\mathrm{Ad}}=e^{i \phi_{2}}$, with $\phi_{1}$ and $\phi_{2}$ their phases. For $\Delta \phi=\phi_{2}-\phi_{1}=\pi / 2$, we obtain Figure 7 . The parameter $\gamma$ represents the round-trip dephasing of the resonator given as $\gamma=2 \pi p_{\mathrm{opt}}\left(\Delta \lambda / \lambda^{2}\right)$, where $p_{\mathrm{opt}}=(2 \pi R+$ 2L) $n_{\text {eff }}$ is the optical path of the resonator, $\Delta \lambda$ the wavelength shift from a racetrack resonance, and $n_{\text {eff }}$ the effective index (at resonance wavelength) of the waveguides (see Appendix A).

The $\gamma$ dependencies of the Drop and Through signals are asymmetric. This is the manifestation of the Fano resonance 

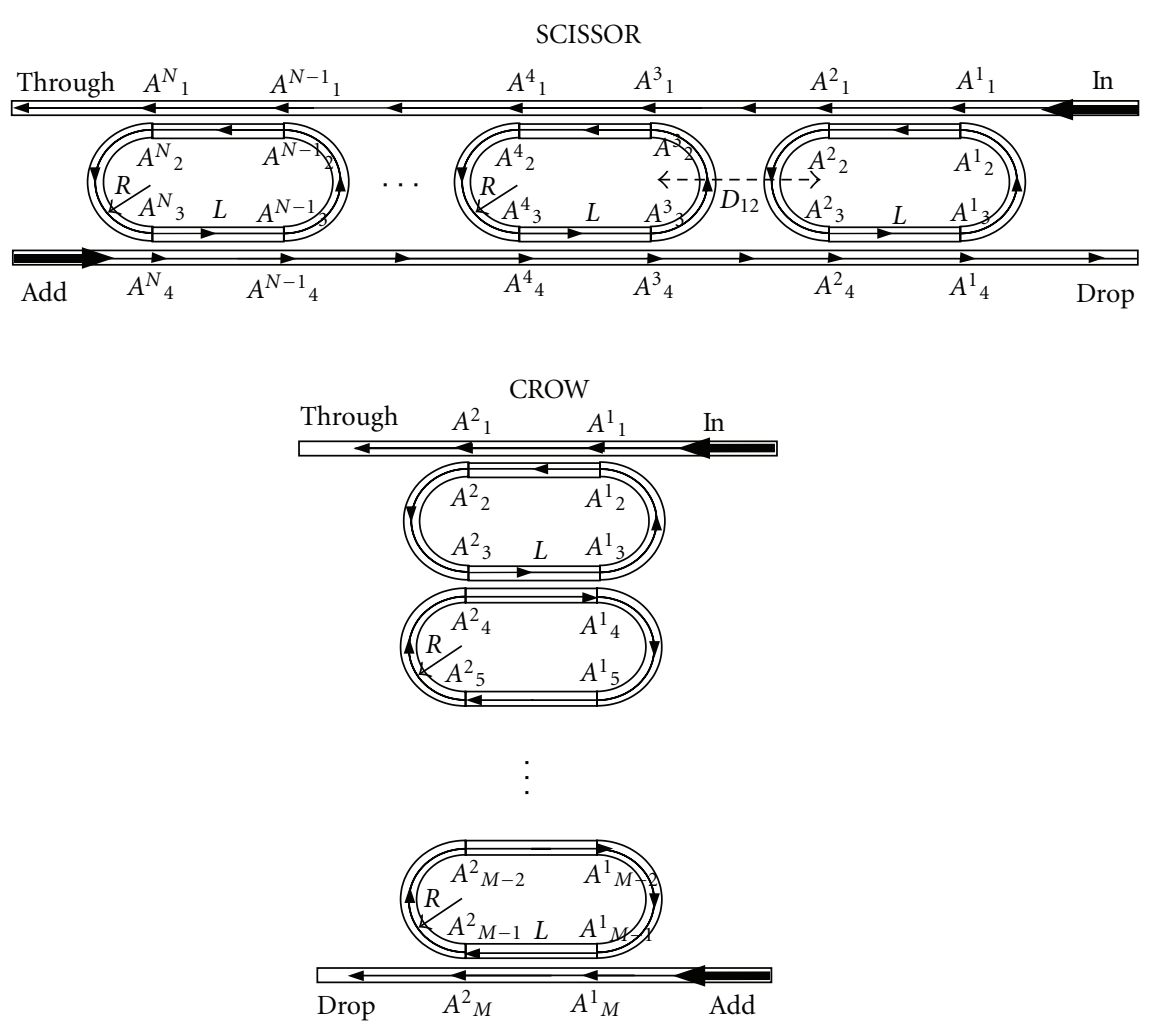

FIGURE 8: The SCISSOR and CROW resonant structures.

that arises from the interference between the resonant modes in the racetrack, where a narrow band superimposes on the waveguide flat spectral background $[23,24]$.

Devices where two phase modulated incoming signals are injected can be used for light switching functions. In this case, a high extinction ratio is obtained. For instance, at $\gamma= \pm 0.6$, in Figure 7 , the drop and through signals have a maximum or vanish (the maximum intensity does not reach 2 because bent losses are considered). Now, if $\Delta \phi$ is changed from $\pi / 2$, as in Figure 7 , to $3 / 2 \pi$, a similar spectrum is found with the two signals changed (the black solid line would go into the red dashed line). This means that, by phase modulation, the signal output can be switched in the Through port or in the Drop port.

It is possible to extend this interferometric switching functions to a parallel or serial chain of coupled resonators, that is, in CROW or SCISSOR, Figure 8.

To model a SCISSOR, we introduce an external propagation matrix $\mathbf{Q}^{j}$ connecting the $j$ th to the $(j+1)$ th resonator

$$
\left(\begin{array}{c}
A_{1}^{j+1} \\
A_{4}^{j+1}
\end{array}\right)=\mathbf{Q}^{j}\left(\begin{array}{l}
A_{1}^{j} \\
A_{4}^{j}
\end{array}\right)=\left(\begin{array}{cc}
e^{i \beta D^{j j+1}} & 0 \\
0 & e^{-i \beta D^{j j+1}}
\end{array}\right)\left(\begin{array}{c}
A_{1}^{j} \\
A_{4}^{j}
\end{array}\right),
$$

where $D^{j j+1}$ is the distance which separates the $j$ th to $(j+$ 1)th resonator. Then, (30) can be generalized to the SCISSOR structure with a scattering matrix $\mathbf{S}_{\mathbf{h}}$ :

$$
\left(\begin{array}{c}
A_{\mathrm{Th}} \\
A_{D}
\end{array}\right)=\mathbf{S}_{\mathbf{h}}\left(\begin{array}{c}
A_{\mathrm{In}} \\
A_{\mathrm{Ad}}
\end{array}\right)=\mathbf{S}^{N} \mathbf{Q}^{N-1} \mathbf{S}^{N-1} \cdots \mathbf{Q}^{1} \mathbf{S}^{1}\left(\begin{array}{c}
A_{\mathrm{In}} \\
A_{\mathrm{Ad}}
\end{array}\right)
$$

For the CROW, we simply generalize (29) to

$$
\left(\begin{array}{c}
A_{\mathrm{Ad}} \\
A_{D}
\end{array}\right)=\mathbf{T}^{N} \cdots \mathbf{T}^{2} \mathbf{T}^{1}\left(\begin{array}{c}
A_{\mathrm{In}} \\
A_{\mathrm{Th}}
\end{array}\right)
$$

where $\mathbf{T}^{k}(k=1, \ldots, N)$ is the transfer matrix for the $k$ th resonator and is given by (28). From this we obtain, the scattering matrix $\mathbf{S}_{\mathbf{v}}$

$$
\left(\begin{array}{c}
A_{\mathrm{Th}} \\
A_{D}
\end{array}\right)=\mathbf{S}_{\mathbf{v}}\left(\begin{array}{c}
A_{\text {In }} \\
A_{\mathrm{Ad}}
\end{array}\right)
$$

Figure 9 shows a spectrum of a SCISSOR made of a chain of eight racetrack resonators. Figure 9(a) represents the spectrum when only one signal is injected: high-order filter flat-box spectrum appears. When a second signal is injected at the Add port with the same amplitude but with a phase difference $\Delta \phi=\pi / 2$ with respect to the signal injected in the In port, Figure 9(b) is obtained. We call this situation, where we have two input signals, the double bus situation. The point $\gamma= \pm 1.5$ can be used to switch the signal from the Through to the Drop port by controlling the phase at the Add signal. Moving the Add signal phase from $\pi / 2$ to $3 / 2 \pi$ switches the output from the Through to the Drop port. Possibly, a wavelength band can be switched by using the $\gamma= \pm 1.75$ point, though with low extinction rates. Therefore, with this respect, the SCISSOR geometry presents limited advantages over the single resonator case of Figure 7.

An eight racetrack CROW structure has a behavior shown in Figure 10. As in the previous case, the left and right 


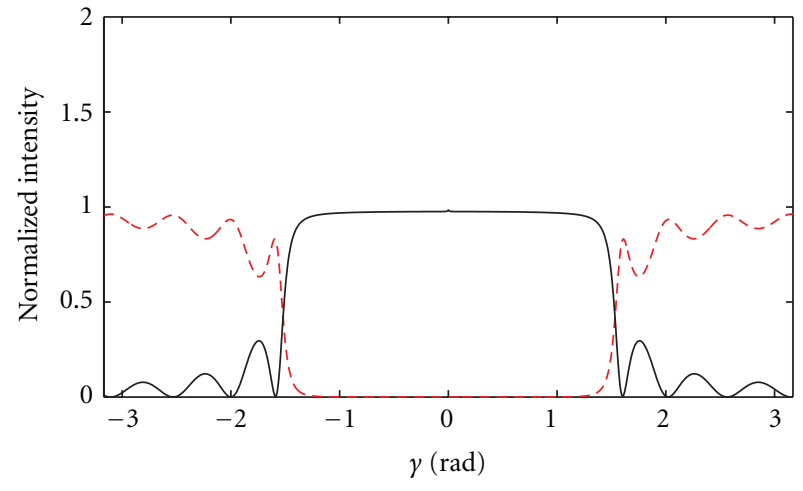

(a)

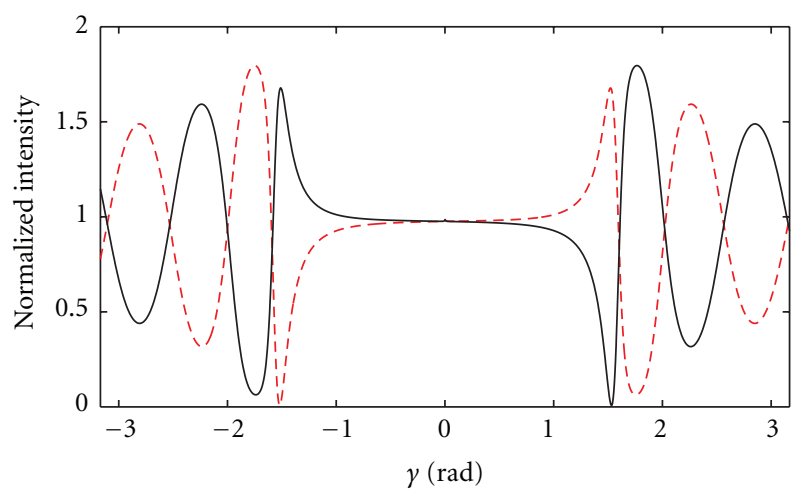

(b)

FIgURE 9: The single (a) and dual-bus (b) eight resonator SCISSOR spectrum for $\Delta \phi=\pi / 2$. (Drop: black solid line, Through: red dashed line).

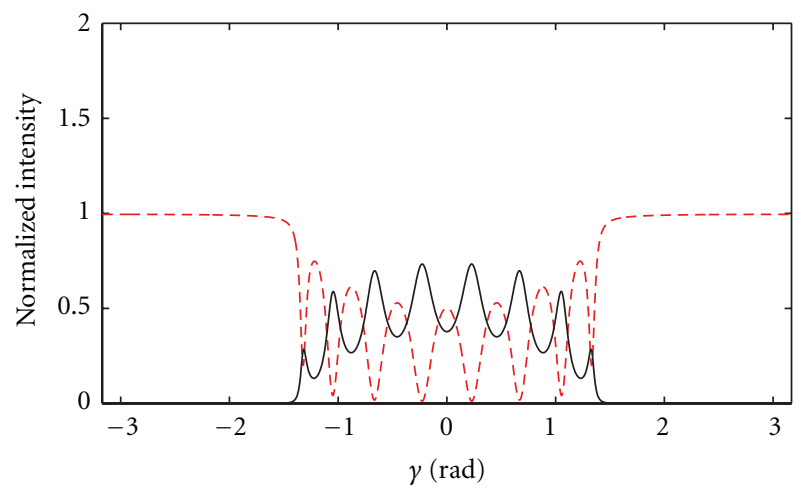

(a)

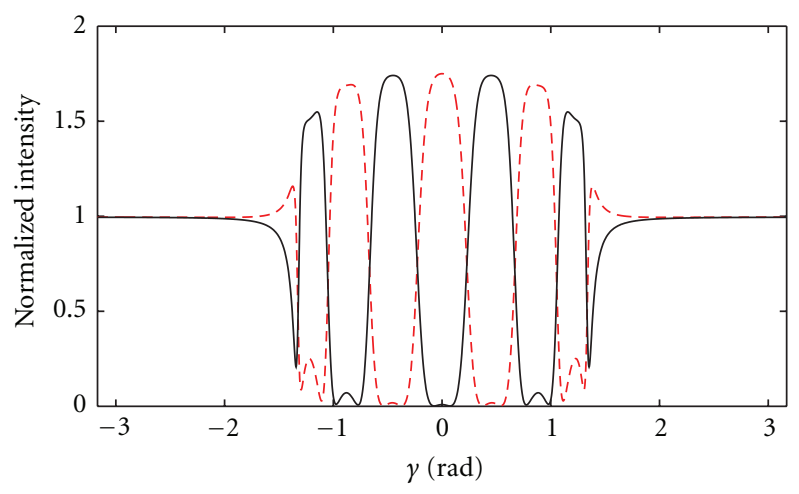

(b)

Figure 10: The single (a) and dual-bus (b) eight resonator CROW spectrum for $\Delta \phi=\pi / 2$. (Lines as in Figure 9).

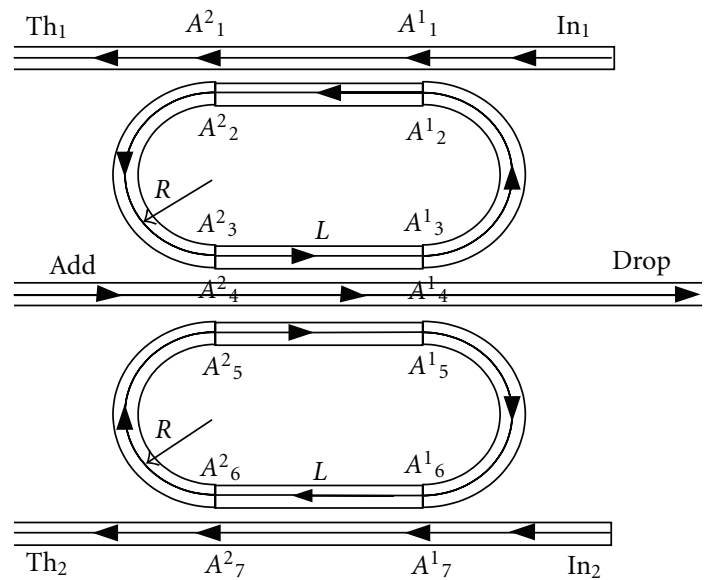

FIGURE 11: The resonator pair ds-coupler switch.

graphs represent the single- and double-bus spectra for the same dephasing. CROW are very different from SCISSOR: given a $N$ resonator CROW, the single bus device has $N$ Drop resonances (Figure 10(a)) while the double bus device has $N-1$ bands when a $\Delta \phi=\pi / 2$ is considered (Figure 10(b)).
Also, in this case, for a dephasing of $\Delta \phi=(3 / 2) \pi$, one can flip the Through $\leftrightarrow$ Drop port outputs. Not all bands have the same extinction efficiency, only the central ones exhibit an almost ideal on-off. The CROW has the advantage with respect to the SCISSOR and single resonator that it allows band routing or it can be used as an optical interleaver, that is, as a device that separates a set of channels into two sets, routing them separately towards the Drop and Through ports. The width of each band depends on the structural parameters.

\section{Three-Bus Resonator Interferometric Switch}

A further interesting situation is to model a structure like that of Figure 11. It shows a single pair of racetrack resonators coupled to three-bus waveguides. This interferometric switch works as follows: the input signals $\mathrm{In}_{1}$ and $\mathrm{In}_{2}$ are tuned to a resonance wavelength and have the same amplitude. Then, depending on their relative phase, they interfere constructively or destructively in the Drop waveguide. In the former case, the signal is expected to couple into the Drop port, while, in the latter case, the signal is transmitted to the Through ports. Experiments demonstrate this principle $[11,12]$. 


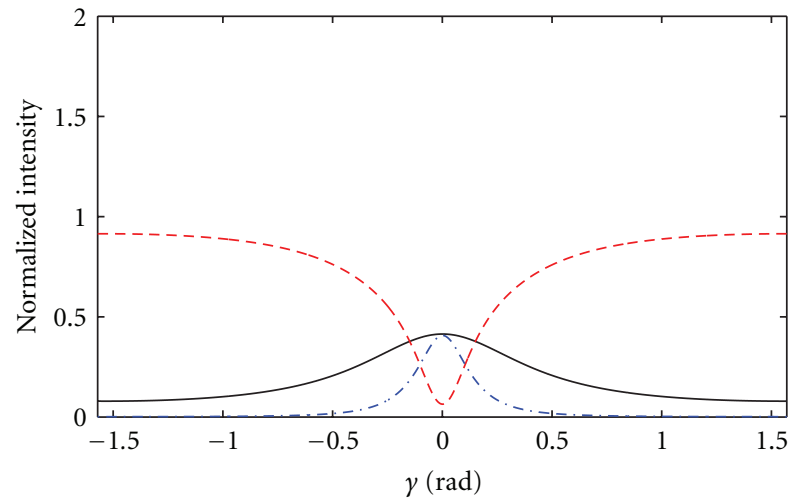

(a)

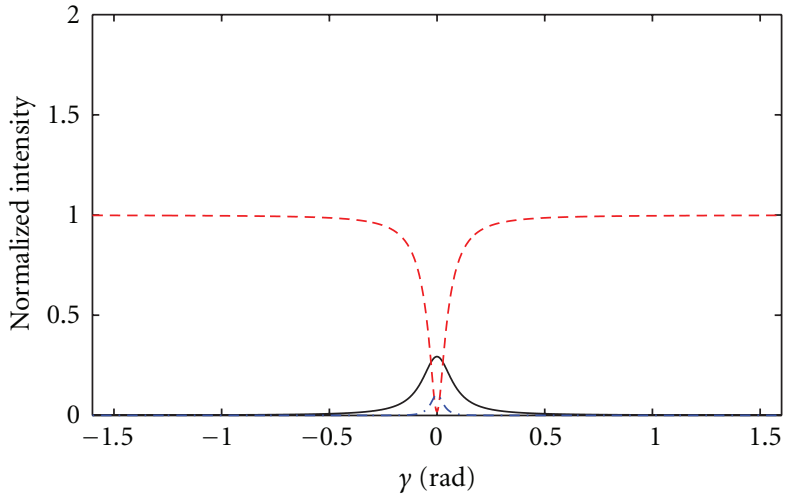

(b)

FIGURE 12: Spectral response of the device in Figure 11, with $A_{\operatorname{In}_{1}}=1, A_{\mathrm{Ad}}=0, A_{\operatorname{In}_{2}}=0, L=10 \mu \mathrm{m}$ (a), and $L=3 \mu \mathrm{m}$ (b) (Drop port: black solid line, Through1 port: red dashed line, Through2 port: blue dotted-dashed line).

In this section, we model the device as a three-bus device, that is, we drive it with three input signals whose phase is independently controlled. To relate the known signals $A_{1}^{1}=$ $A_{\mathrm{In}_{1}}, A_{4}^{2}=A_{\mathrm{Ad}}, A_{7}^{1}=A_{\mathrm{In}_{2}}$ to the unknown $A_{1}^{2}=A_{\mathrm{Th}_{1}}$, $A_{4}^{1}=A_{D}$, and $A_{7}^{2}=A_{\mathrm{Th}_{2}}$ (Figure 11), we use a general transfer matrix $\mathbf{T}$ :

$$
\left(\begin{array}{c}
A_{\mathrm{Th}_{1}} \\
A_{D} \\
A_{\mathrm{Th}_{2}}
\end{array}\right)=\left(\begin{array}{lll}
T_{11} & T_{21} & T_{31} \\
T_{21} & T_{22} & T_{21} \\
T_{31} & T_{21} & T_{11}
\end{array}\right)\left(\begin{array}{c}
A_{\mathrm{In}_{1}} \\
A_{\mathrm{Ad}_{1}} \\
A_{\mathrm{In}_{2}}
\end{array}\right)=\mathrm{T}\left(\begin{array}{c}
A_{\mathrm{In}_{1}} \\
A_{\mathrm{Ad}_{1}} \\
A_{\mathrm{In}_{2}}
\end{array}\right),
$$

whose elements are reported in Appendix B. The device (and therefore $\mathbf{T})$ is symmetric: the paths $\mathrm{In}_{1} \rightarrow$ Through ${ }_{1}, \mathrm{In}_{1} \rightarrow$ Drop, $\operatorname{In}_{1} \rightarrow$ Through 2 are equivalent to the paths $\operatorname{In}_{2} \rightarrow$ Through ${ }_{2}, \mathrm{In}_{2} \rightarrow$ Drop, $\mathrm{In}_{2} \rightarrow$ Through $_{1}$.

Figure 12 shows the spectral response of the device when used in single bus configuration, that is, only one input signal is injected $\left(A_{\mathrm{In}_{1}}=1, A_{\mathrm{Ad}}=0, A_{\mathrm{In}_{2}}=0\right)$, for two coupling section lengths of $L=10 \mu \mathrm{m}$ and $L=3 \mu \mathrm{m}$. The drop signal (black line) shows a typical resonant behavior with a sharper feature for the $L=3 \mu \mathrm{m}$ device than for the $L=10 \mu \mathrm{m}$ device because of its lower coupling efficiency. It is also interesting to note that the crosstalk is much higher for $L=10 \mu \mathrm{m}$ than for $3 \mu \mathrm{m}$. At resonance, the power is almost equally distributed between the Drop and Through 2 ports.

Figure 13 shows the case of a device operated in a duals bus configuration. We used $L=10 \mu \mathrm{m}$ for the coupling section. The input signals have the same amplitude and a phase difference of $\Delta \phi=0, \pi / 4,3 / 4 \pi, \pi$ for Figures $13(\mathrm{a})$, 13(b), 13(c), 13(d), respectively. When the two input signals are in phase, and the wavelength is resonant with the two racetracks, then most of the power is transferred to the Drop port. This situation was experimentally confirmed in $[11,12]$.

The Through ports will not exhibit complete extinction due to crosstalk effects (Figure 13(a)). Similarly, when the two input signals are in antiphase (Figure 13(d)), a complete destructive interference is achieved in the Drop waveguide and no Drop signal is observed. Most of the power goes straight in the Through ports. Note that the minima at resonance in the Through signals are due to the resonator round trip losses. With no dephasing, the Through signal minima coincide, with the Drop signal maximum at resonance $\lambda_{0}$ (Figure 13(a)). In the other case, the Through signals show the behaviors of a Fano resonance (Figures 13(b) and 13(c)). When there is a phase shift between the input signals, the Through signals show characteristics of Fano resonance with a different minimum at $\lambda_{F}=\lambda_{0} \pm 0.8 \mathrm{~nm}$. This occurs already for a small $\Delta \phi=\pi / 10$. Moreover, the Through signal resonance depth depends on the phase shift and vanishes almost completely at $\Delta \phi=\pi / 4$ (Figure 13(b)). This is potentially useful to build interferometric switching devices with good on-off extinctions.

To highlight the phase shift dependence, we repeated the calculations at the resonant or at the Fano wavelengths $\left(\lambda_{0}\right.$ or $\lambda_{F}$, resp.) by varying $\Delta \phi$. At the same time, we varied the intensity of the input signals. The results are shown in Figure 14 for $\lambda_{0}$ and in Figure 15 for $\lambda_{F}$, for $\left|A_{\operatorname{In}_{2}}\right|^{2}=\delta \times$ $\left|A_{\mathrm{In}_{1}}\right|^{2}$ with $\delta=(1,0.75,0.25,0)$. Several features can be observed. First, for perfect input balance (Figure 14(a) and Figure 15(a)), the Drop signal goes to zero at $\Delta \phi=\pi$, while the Through signals vanish only for $\lambda_{F}$ and not for $\lambda_{0}$. For this reason, interferometric switching is at $\lambda_{F}$ than at $\lambda_{0}$. Second, for $\lambda_{F}$ (Figure 15), the three output signals (Drop, Through and Through ${ }_{2}$ ) vanish for three different values of $\Delta \phi: \Delta \phi=$ $\pi / 4$ the Through $2, \Delta \phi=\pi$ the Drop, and $\Delta \phi=7 / 4 \pi$ the Through $_{1}$. Third, the transmission of the input signals to the through ports is more effective for $\lambda_{F}$ than for $\lambda_{0}$, which might be useful for practical purposes. Fourth, from Figures 14 and 15, it is clear that an interferometric switch device must be robust against input signal imbalance.

Similar features are obtained for a device with $L=3 \mu \mathrm{m}$. The main difference is that $\lambda_{F}$ is more near to $\lambda_{0}$ which makes difficult the use of the Fano concepts for interferometric routing.

A further extension of the ds-router concept is a double SCISSOR structure as shown in Figure 16. To model it, we transform $\mathbf{T}$ (35) into the "scattering matrix" $S$ such that

$$
\left(\begin{array}{c}
A_{\mathrm{Th}_{1}} \\
A_{\mathrm{Ad}} \\
A_{\mathrm{Th}_{2}}
\end{array}\right)=\mathrm{S}\left(\begin{array}{c}
A_{\mathrm{In}_{1}} \\
A_{D} \\
A_{\mathrm{In}_{2}}
\end{array}\right)
$$




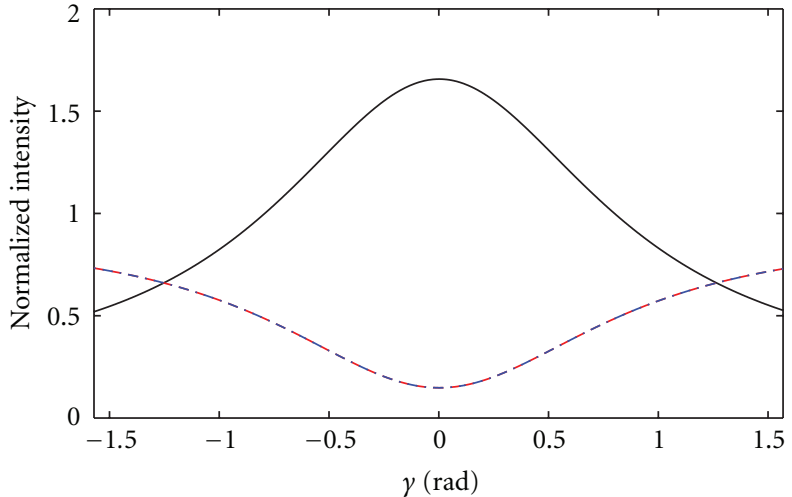

(a)

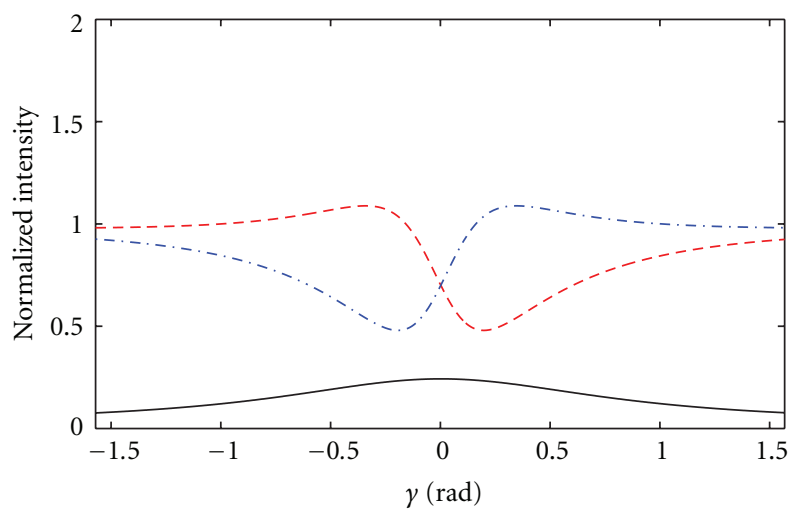

(c)

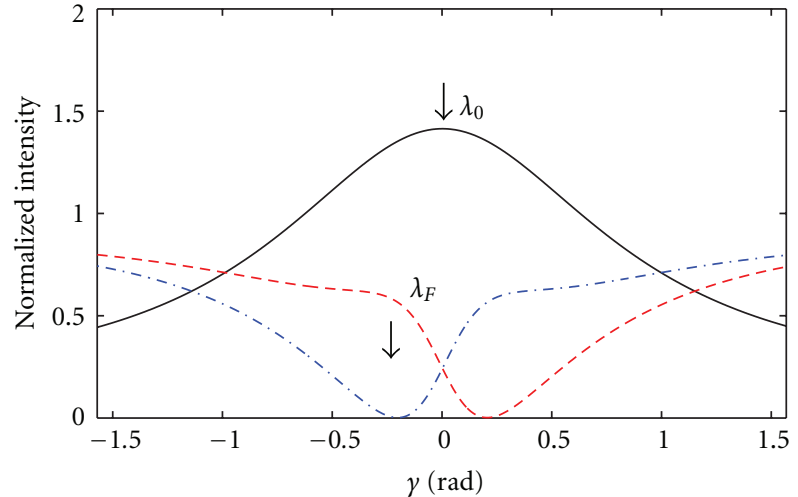

(b)

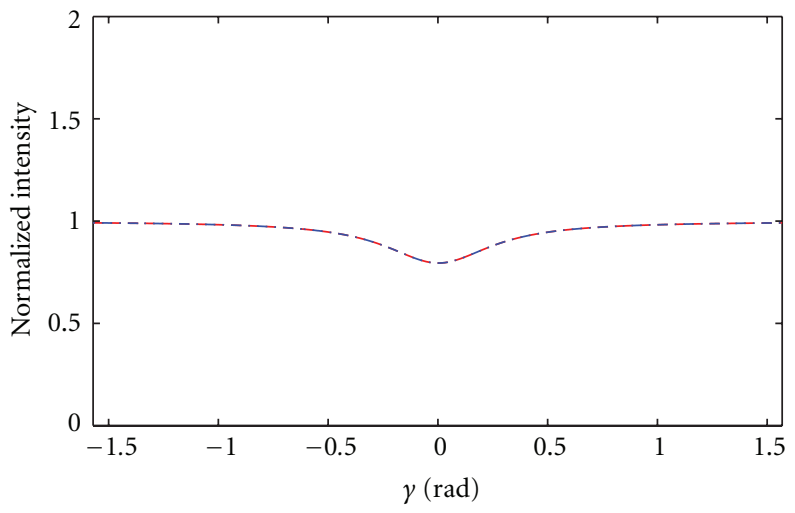

(d)

FIGURE 13: Spectral response of the device in Figure 11, with $A_{\operatorname{In}_{1}}=\left(1, e^{i(\pi / 4)}, e^{i(3 / 4) \pi}, e^{i \pi}\right)((\mathrm{a}),(\mathrm{b}),(\mathrm{c}),(\mathrm{d})$, resp. $), A_{\mathrm{Ad}}=0, A_{\operatorname{In} 2}=1$. (Lines as in Figure 12.)

After simple algebraic manipulation, one finds

$$
\mathbf{S}=\frac{1}{T_{22}}\left(\begin{array}{ccc}
T_{11} T_{22}-T_{12} T_{21} & T_{12} & T_{31} T_{22}-T_{12} T_{21} \\
-T_{21} & 1 & -T_{21} \\
T_{31} T_{22}-T_{12} T_{21} & T_{12} & T_{11} T_{22}-T_{12} T_{21}
\end{array}\right),
$$

where the lower indexes indicate the row $\times$ column element of $\mathrm{T}$.

Then, by introducing an external propagation matrix $\mathbf{Q}^{k}$ which connects the Through and Add ports of the $k$ th pair of resonators with the In and Drop ports of the $(k+1)$ th pair as

$$
\begin{array}{r}
\left(\begin{array}{c}
A_{\mathrm{In}_{1}^{k+1}} \\
A_{D^{k+1}} \\
A_{\mathrm{In}_{2}^{k+1}}
\end{array}\right)=\mathbf{Q}^{\mathbf{k}}\left(\begin{array}{c}
A_{\mathrm{Th}_{1}^{k}} \\
A_{\mathrm{Ad}^{k}} \\
A_{\mathrm{Th}_{2}^{k}}
\end{array}\right)=\left(\begin{array}{ccc}
e^{i \beta D^{k}} & 0 & 0 \\
0 & e^{-i \beta D^{k}} & 0 \\
0 & 0 & e^{i \beta D^{k}}
\end{array}\right)\left(\begin{array}{c}
A_{\mathrm{Th}_{1}^{k}} \\
A_{\mathrm{Ad}^{k}} \\
A_{\mathrm{Th}_{2}^{k}}^{k}
\end{array}\right) \\
(k=1, \ldots, N),
\end{array}
$$

we can express the general equations for the device of Figure 16 with a total transfer matrix $\mathbf{S}_{\mathrm{Tot}}$ :

$$
\left(\begin{array}{c}
A_{\mathrm{Th}_{1}^{N}} \\
A_{\mathrm{Ad}^{N}} \\
A_{\mathrm{Th}_{2}^{N}}
\end{array}\right)=\mathbf{S}_{\mathrm{Tot}}\left(\begin{array}{c}
A_{\mathrm{In}_{1}^{1}} \\
A_{D^{1}} \\
A_{\mathrm{In}_{2}^{1}}
\end{array}\right)=\mathbf{S}^{N} \mathbf{Q}^{N-1} \mathbf{S}^{N-1} \cdots \mathbf{Q}^{1} \mathbf{S}^{1}\left(\begin{array}{c}
A_{\mathrm{In}_{1}^{1}} \\
A_{D^{1}} \\
A_{\mathrm{In}_{2}^{1}}
\end{array}\right) .
$$

And, finally, after inverting $\boldsymbol{S}_{\text {Tot }}(37)$, we get

$$
\left(\begin{array}{c}
A_{\operatorname{Th}_{1}^{N}} \\
A_{D^{1}} \\
A_{\mathrm{Th}_{2}^{N}}
\end{array}\right)=\mathbf{T}_{\mathrm{Tot}}\left(\begin{array}{c}
A_{\mathrm{In}_{1}^{1}} \\
A_{\mathrm{Ad}^{N}} \\
A_{\mathrm{In}_{2}^{1}}
\end{array}\right)
$$

which is the generalized version of (35).

Figure 17 shows the spectral response for the 1,2,4, and 8 pair SCISSOR interferometric switch (Figures 17(a), 17(b), 17(c), 17(d), resp.) with only one input signal. The $(N-1)$ central dips are not due to fabrication imperfections $[11,12]$ but are intrinsic features due to the existence of the central Drop waveguide between the two resonator chains. The system behaves like a single SCISSOR row with asymmetric gaps which introduce a correspondent asymmetric phase shift at each resonator gap leading to CRIT-like features. It is in this spectral region, near the center of the resonance band, where light couples more efficiently into the resonators and travels repeatedly through them producing slow light effects. For this reason, it is also in this central CRITlike region where most of the attenuation occurs due to radiation and roughness losses on the resonator bent. Note how, aside from the flat-box spectrum, despite strong input imbalance, the two Through ports response (red dashed and blue dotted-dashed lines) tend to merge together inside the mode's spectral range with increasing the number of 


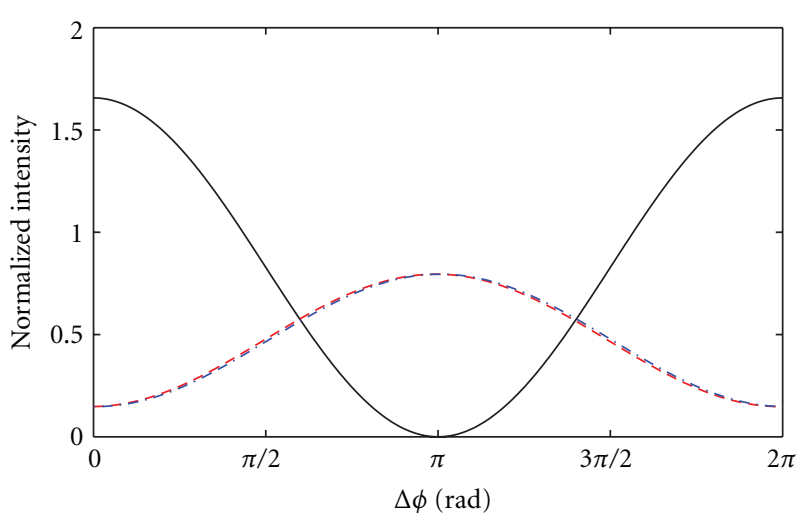

(a)

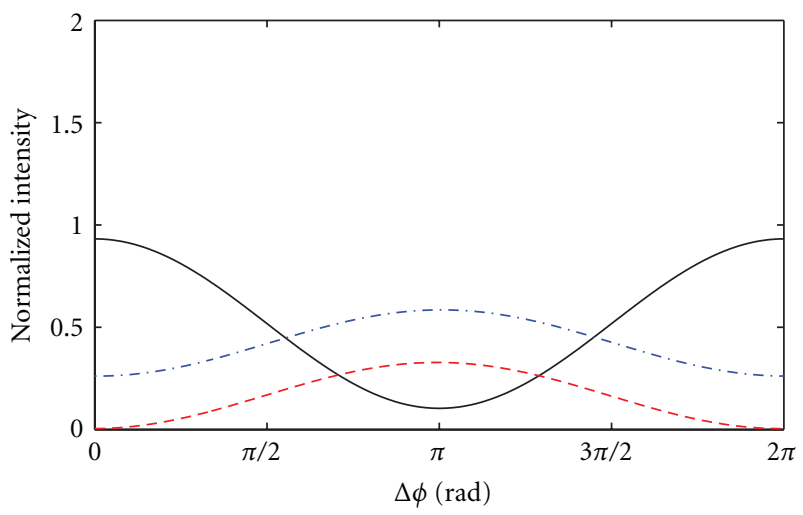

(c)

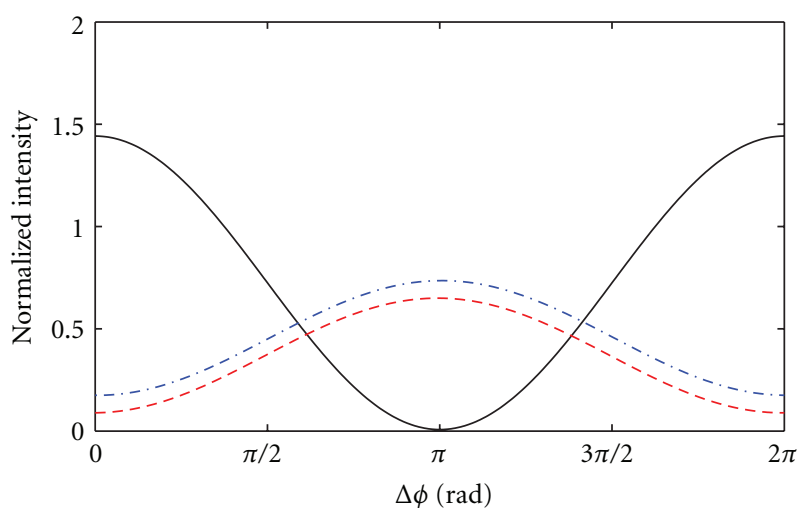

(b)

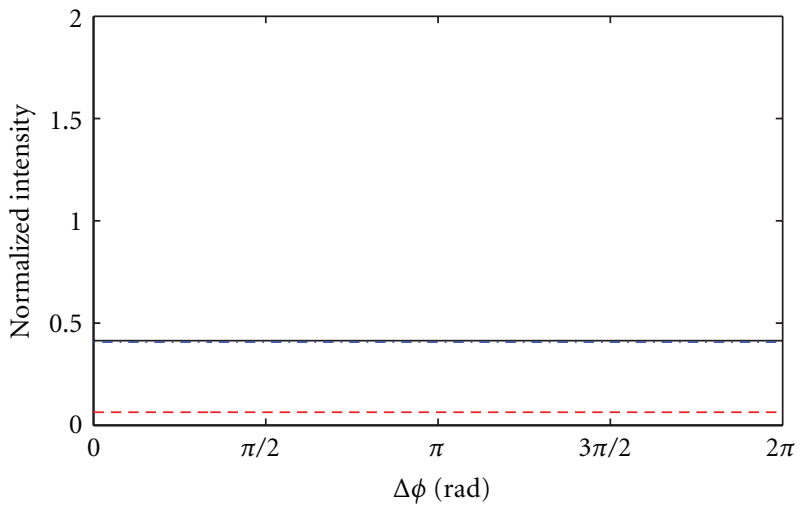

(d)

Figure 14: Phase response of the device in Figure 11 at $\lambda_{0}$, with $A_{\operatorname{In}_{1}}=e^{i \Delta \Phi}, A_{\mathrm{Ad}}=0,\left|A_{\operatorname{In}_{2}}\right|^{2}=(1,0.75,0.25,0)((\mathrm{a})$, (b), (c), (d), resp.) (Lines as in Figure 12.)

resonator pairs. This is because in an $N$-pair SCISSOR the input signals couple to the various resonator pairs, and, while they are propagating along the sequence, their power is progressively balanced due to the large crosstalk. This robustness of SCISSOR devices against input imbalances could have practical advantages where a power injection is uncertain or difficult to tailor.

Figure 18 illustrates the use of a dual bus SCISSOR interferometric switch with two equal input signals. Increasing the number of resonator, the response gets a flat-box shape and a good rejection of the input signal at the two Through ports is obtained.

Figure 19 shows the effect of a phase shift between the two input signals. As $\Delta \phi$ increases, the Drop signal decreases and vanishes for $\Delta \phi=\pi$. The Through signals are coincident within the stop-band and differ outside this wavelength region when $\Delta \phi \neq 0$.

We studied also the effect of an imbalance between the two input signals. A phase sweep at $\gamma=1$ for four different intensities is shown in Figure 20. Contrary to the single pair interferometric switch affected by Fano resonance asymmetries and unequal Through port signal intensities along the phase sweep, the $2 \times 8$ SCISSOR interferometric switch flattens out the differences on the Through signals and shows its robustness against imbalances.

\section{Three-Bus Interferometric Switch with Three Input Signals}

Another potentially interesting application is to operate the ds-coupler interferometric switch of Figure 11 with three input signals, that is, to use the Add port as a third input port. Figure 21 shows its spectral response when $A_{\mathrm{In}_{1}}=e^{i \phi_{1}}$, $A_{\mathrm{Ad}}=e^{i \phi_{\mathrm{Add}}}, A_{\mathrm{In}_{2}}=e^{i \phi_{2}}$. Figure 21(a) shows the case of all three equal input phases $\left(\left(\phi_{1}, \phi_{\text {Add }}, \phi_{2}\right)=(0,0,0)\right)$ : the Drop and the two Through signals are equal with slight differences caused by the bending losses. Figure 21(b) reproduces the case $\left(\phi_{1}, \phi_{\text {Add }}, \phi_{2}\right)=(0, \pi / 4, \pi / 2)$ : at the resonance wavelength, the two Through signals are high while the Drop signals are low. The Through signal resonances are Fano resonances. Figure 21(c) represents the alternative case where only a $\pi$-shift on the Add signal is applied $\left(\left(\phi_{1}, \phi_{\text {Add }}, \phi_{2}\right)=\right.$ $(0, \pi, 0))$ : the Drop signal maximizes at resonance, while the two Through signals are low. Figure 21(d) shows the case when a $\pi$-shift is applied to both the Add and one Input signal $\left(\phi_{1}, \phi_{\text {Add }}, \phi_{2}\right)=(0, \pi, \pi)$ : all the input signals are addressed to the Through 1 port. The symmetric case where the signals are directed to the Through ${ }_{2}$ port is obtained by applying the same phase shift to the other Input signal.

This three input signal configuration allows using the Add signal as a control signal to drive the interferometric 


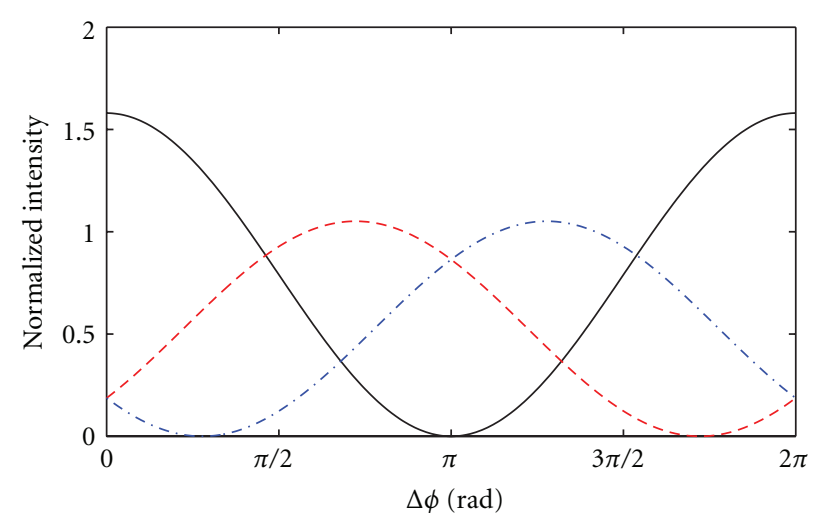

(a)

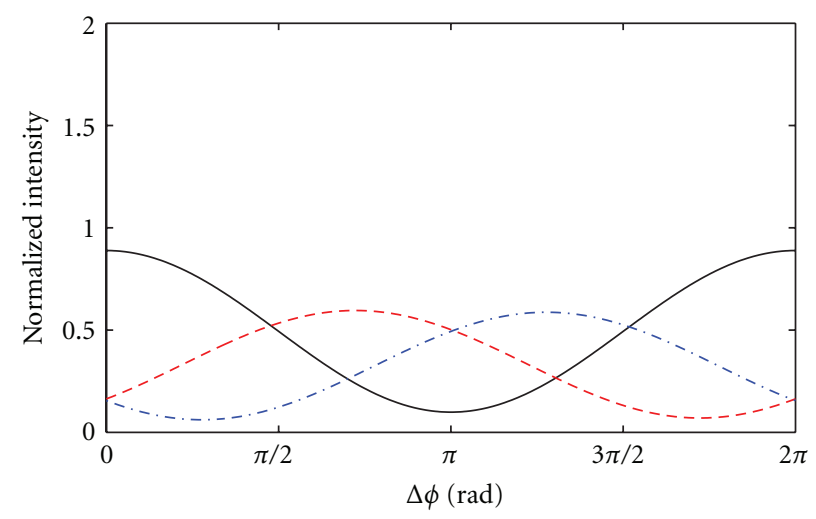

(c)

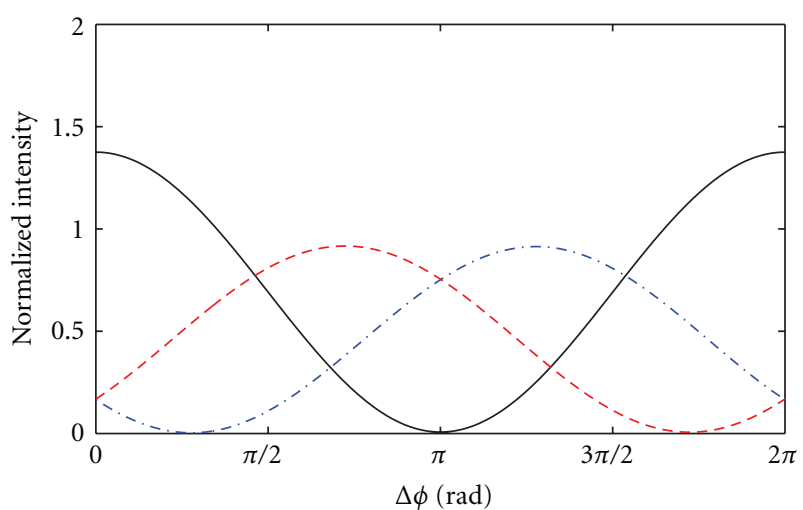

(b)

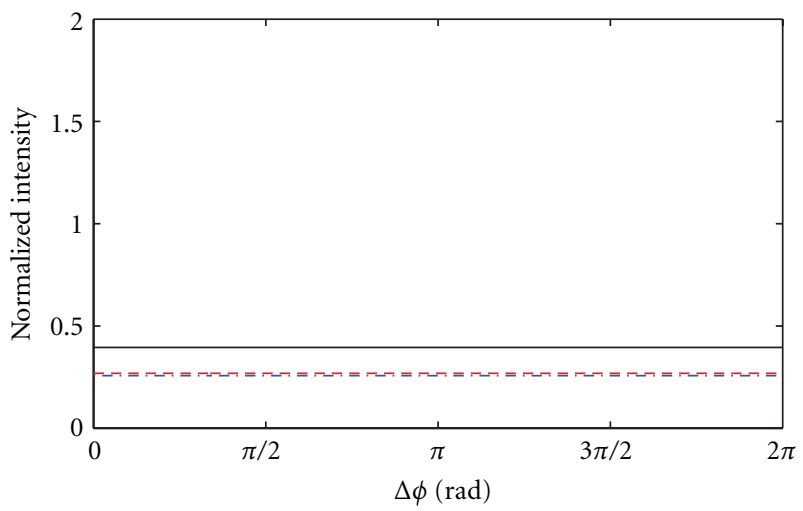

(d)

Figure 15: Phase response of the device in Figure 11 at $\lambda_{F}$, with $A_{\operatorname{In}_{1}}=e^{i \Delta \Phi}, A_{\mathrm{Ad}}=0,\left|A_{\operatorname{In}_{2}}\right|^{2}=(1,0.75,0.25,0)((\mathrm{a}),(\mathrm{b}),(\mathrm{c}),(\mathrm{d})$, resp.). (Lines as in Figure 12.)

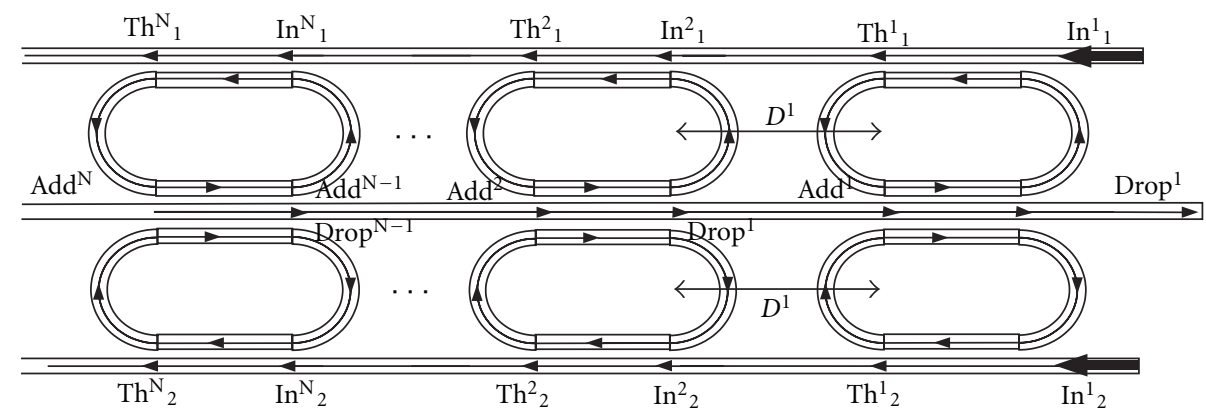

FIGURE 16: The $2 \times N$ SCISSOR phase switch.

switch to different functionalities (compare Figure 13 with Figure 21). In fact, if the phase of the Add signal is changed from 0 to $\pi$, the Drop signal is switched from 1 to 3 (in absence of bending losses); while, if the phase of one of the input is changed from 0 to $\pi$ and $\phi_{\text {Add }}=\pi$, one can switch off and on the Through signals. Many other sorts of combinations in phase and intensity at the Input ports, resonator numbers, and overall resonant device geometry for phase switching applications could be imagined in the most diverse configurations.

\section{Conclusions}

A three-bus waveguide multiple resonator-based interferometric switching device was discussed which is able to route light over a broad range of wavelengths by phase 


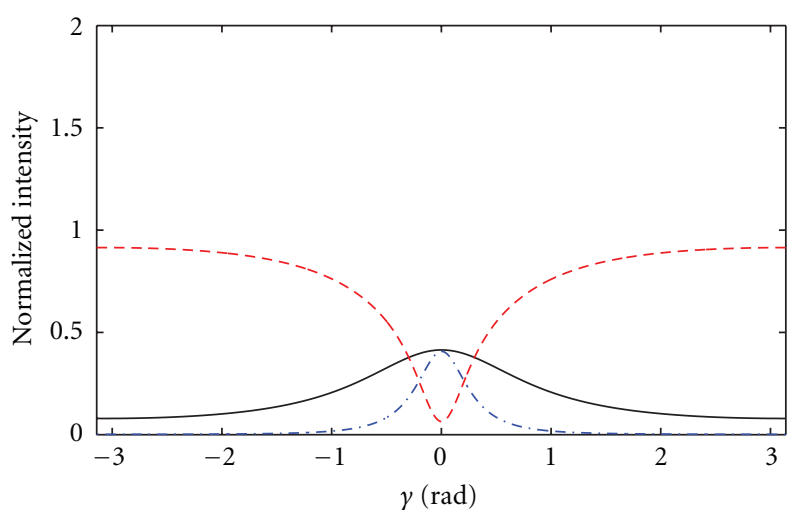

(a)

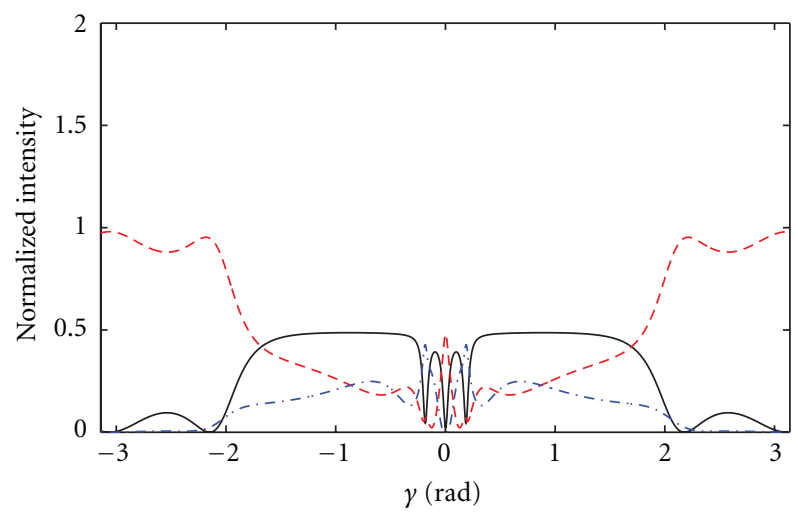

(c)

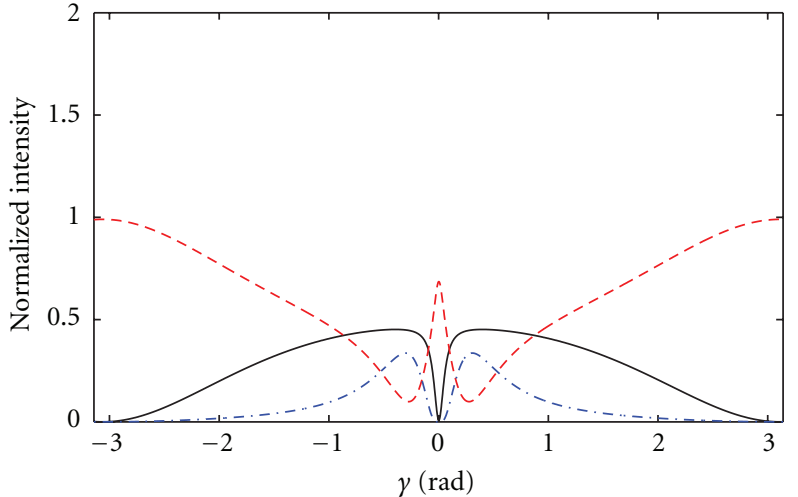

(b)

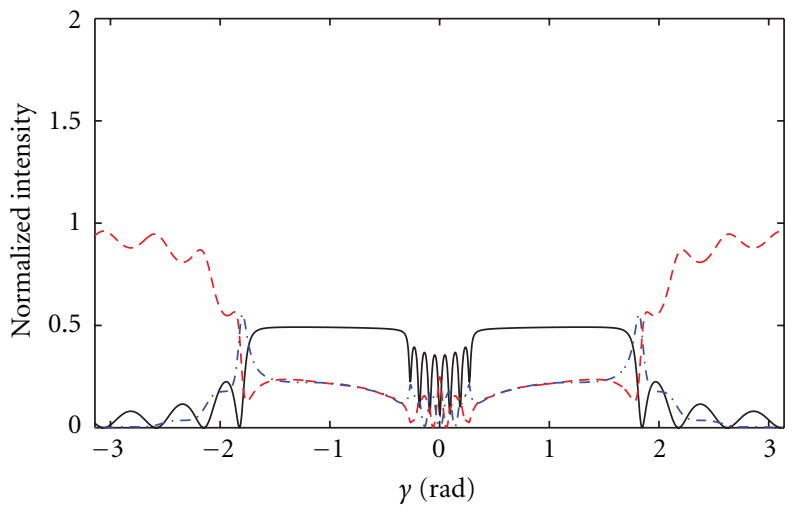

(d)

FIGURE 17: Spectral response of the SCISSOR switch with 1, 2, 4, 8 pairs of resonators ((a), (b), (c), (d), resp.) and inputs $A_{\text {In }_{1}}=1, A_{\mathrm{Ad}}=0$, $A_{\text {In }_{2}}=0$. (Lines as in Figure 12.)

modulation. To obtain its behavior, it was necessary to analyze first the performance of the two bus waveguide single resonator and SCISSOR and CROW systems. We showed that extending side coupling and Mach-Zehnder phase switching techniques to multiple waveguides and to chains of resonators could be useful to enhance their switching capabilities. The proposed interferometer device shows the possibility to control a lightwave signal with the phase of another signal. This allows to add a further degree of freedom in designing reconfigurable optical routers and complex networks. Simple examples can be foreseen in single channel switches where the switch redirects the signal in two different channels without absorbing the light (Figure 7), or in a dual channel interleaver where the signal bands can be alternatively directed on one or the other channels by phase control (Figure 10(b)), or in a three channel router where the signal is routed along three different directions by controlling the wavelength and the phase (Figure 13(b)). It is clear, though, that the potentiality of the scheme proposed in this work is not exhausted by these examples and more can be envisaged by optical network specialists. However, we believe that herewith we outlined some proposals which display how this is a field with vast possibilities, hopefully inspiring further investigations of possible applications of novel interferometric switching devices.

\section{Appendices}

\section{A.}

Instead of displaying the spectral response of a resonant structure in terms of the wavelength, it is possible to express it as a resonator round trip dephasing, clearly with the assumption of a nondispersive system. The resonant wavelength of the $m$ th mode, $\lambda_{m}$, is thus in general

$$
\lambda_{m}=\frac{p_{\text {opt }}\left(\lambda_{m}\right)}{m},
$$

with the wavelength-dependent optical path of the racetrack resonator $p_{\text {opt }}=(2 \pi R+2 L) n_{\text {eff }}\left(\lambda_{m}\right), n_{\text {eff }}\left(\lambda_{m}\right)$ the effective index of the resonator waveguides at the $m$ th resonant wavelength, and $m$ the resonance azimutal number. In the case of dispersionless systems, (A.1) is no longer an implicit function, and any two modes at $\lambda_{1}$ and $\lambda_{2}$ are determined solely by the optical path of the resonator $\left(p_{\text {opt }}\left(\lambda_{m}\right)=\right.$ $p_{\text {opt }}$ for all $m$ ) and by the mode azimutal number $m$, as

$$
\lambda_{1}=\frac{p_{\mathrm{opt}}}{m}, \quad \lambda_{2}=\frac{p_{\mathrm{opt}}}{m+1} .
$$

This means that the difference (i.e., the FSR) is

$$
\lambda_{2}-\lambda_{1}=-\frac{p_{\mathrm{opt}}}{m(m+1)}=-\frac{\lambda_{1}}{m+1}=-\frac{\lambda_{1} \lambda_{2}}{p_{\mathrm{opt}}} .
$$




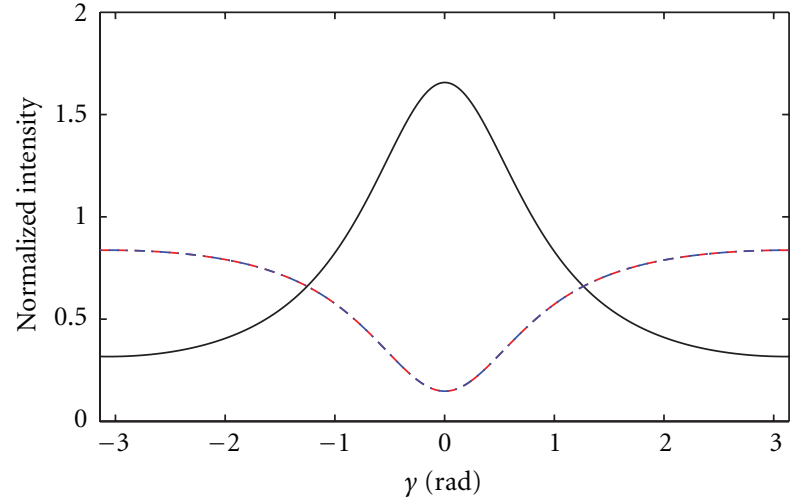

(a)

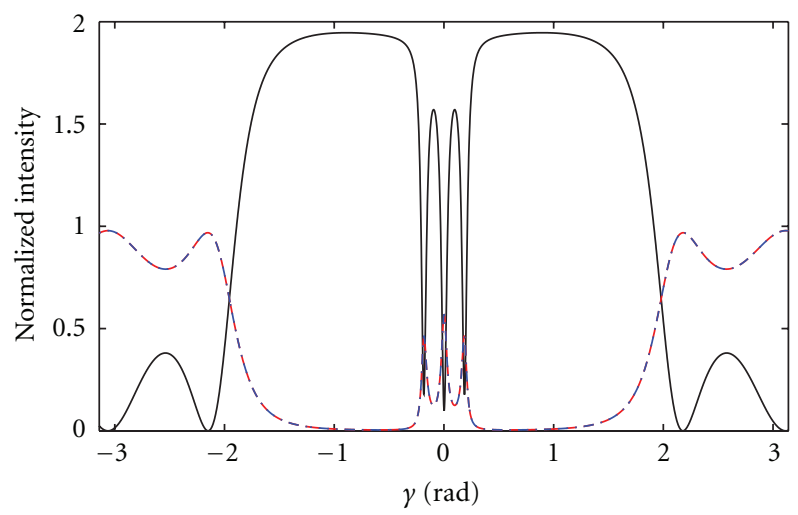

(c)

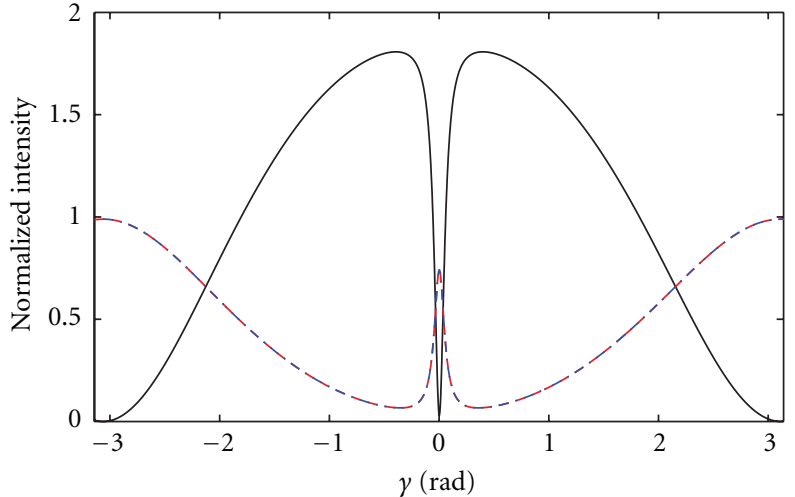

(b)

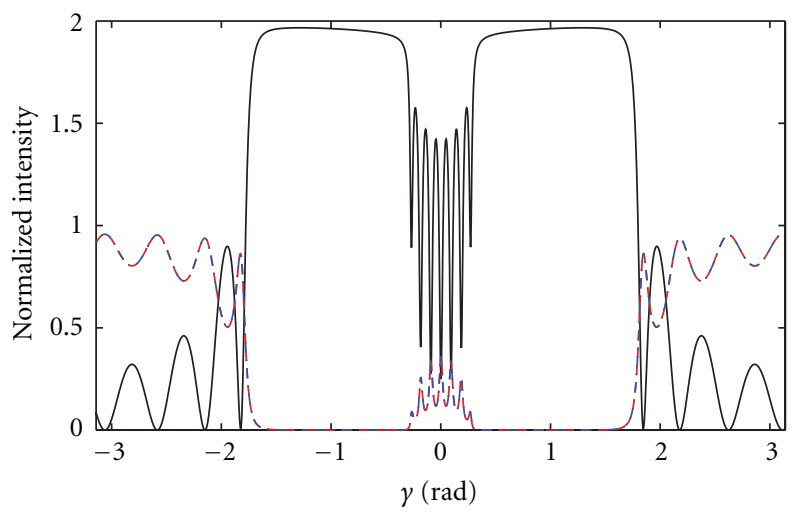

(d)

FIGURE 18: Spectral response of the SCISSOR switch with 1, 2, 4, 8 pairs of resonators ((a), (b), (c), (d), resp.), and inputs $A_{\text {In }_{1}}=1, A_{\mathrm{Ad}}=0$, $A_{\operatorname{In}_{2}}=1$, with same phase. (Lines as in Figure 12.)

Then, the parameter $\gamma$ representing the round-trip dephasing of the resonator can be defined as an "angular deviation" from resonance at some wavelength $\lambda$ as

$$
\lambda=\lambda_{1}+\frac{\gamma}{2 \pi}\left(\lambda_{2}-\lambda_{1}\right)=\lambda_{1}-\frac{\gamma}{2 \pi} \frac{\lambda_{1} \lambda_{2}}{p_{\mathrm{opt}}}
$$

and from which

$$
\gamma=2 \pi p_{\text {opt }} \frac{\lambda_{1}-\lambda}{\lambda_{1} \lambda_{2}} \approx 2 \pi p_{\text {opt }} \frac{\Delta \lambda}{\lambda^{2}} .
$$

B.

In Figure 11, we can relate $\left(A_{3}^{2}, A_{4}^{2}, A_{5}^{2}\right) \rightarrow\left(A_{3}^{1}, A_{4}^{1}, A_{5}^{1}\right)$ through (13) as

$$
\left(\begin{array}{l}
A_{3}^{1} \\
A_{4}^{1} \\
A_{5}^{1}
\end{array}\right)=\mathcal{M}\left(\begin{array}{l}
A_{3}^{2} \\
A_{4}^{2} \\
A_{5}^{2}
\end{array}\right)=\left(\begin{array}{ccc}
t^{\prime} & i \kappa_{D} & -\kappa^{\prime} \\
i \kappa_{D} & t_{D} & i \kappa_{D} \\
-\kappa^{\prime} & i \kappa_{D} & t^{\prime}
\end{array}\right)\left(\begin{array}{l}
A_{3}^{2} \\
A_{4}^{2} \\
A_{5}^{2}
\end{array}\right)
$$

Proceeding in the same way as we have done with matrix $\mathbf{M}$ of (25), we look for the coupling matrix that relates in the interference section the upper to the lower waveguide through the middle one, that is, $\left(A_{3}^{2}, A_{4}^{2}, A_{3}^{1}\right) \rightarrow\left(A_{5}^{2}, A_{4}^{1}, A_{5}^{1}\right)$, and obtain the second transfer matrix $\mathrm{T}^{\mathbf{b}}$ :

$$
\begin{aligned}
& \left(\begin{array}{c}
A_{5}^{2} \\
A_{4}^{1} \\
A_{5}^{1}
\end{array}\right)=\mathbf{T}^{\mathbf{b}}\left(\begin{array}{c}
A_{3}^{2} \\
A_{4}^{2} \\
A_{3}^{1}
\end{array}\right) \\
& =\frac{1}{\kappa^{\prime}}\left(\begin{array}{ccc}
t^{\prime} & i \kappa_{D} & -1 \\
i \kappa_{D}\left(\kappa^{\prime}+t^{\prime}\right) & t_{D} \kappa^{\prime}-\kappa_{D}^{2} & -i \kappa_{D} \\
t^{\prime 2}-\kappa^{\prime 2} & i \kappa_{D}\left(\kappa^{\prime}+t^{\prime}\right) & -t^{\prime}
\end{array}\right)\left(\begin{array}{c}
A_{3}^{2} \\
A_{4}^{2} \\
A_{3}^{1}
\end{array}\right) .
\end{aligned}
$$

Due to a symmetric coupling, $\mathbf{K}$ of (26) can be applied also at $\left(A_{6}^{1}, A_{6}^{2}\right) \rightarrow\left(A_{7}^{1}, A_{7}^{2}\right)$. For the same reason, the same internal propagation matrix of (27) can be applied also at $\left(A_{5}^{2}, A_{5}^{1}\right) \rightarrow\left(A_{6}^{1}, A_{6}^{2}\right)$. Therefore, the third transfer matrix connects $\left(A_{5}^{2}, A_{5}^{1}\right) \rightarrow\left(A_{7}^{1}, A_{7}^{2}\right)$ in the same way as (28) (but with the order of the propagation and coupling matrixes inverted):

$$
\left(\begin{array}{l}
A_{7}^{1} \\
A_{7}^{2}
\end{array}\right)=\mathrm{T}^{\mathrm{KP}}\left(\begin{array}{l}
A_{5}^{2} \\
A_{5}^{1}
\end{array}\right)=\mathbf{K P}\left(\begin{array}{l}
A_{5}^{2} \\
A_{5}^{1}
\end{array}\right) .
$$

To proceed in the inverse direction, that is, from ports $\left(\operatorname{In}_{2}\right.$, Through $\left._{2}\right)$ to $\left(\operatorname{In}_{1}\right.$, Through $\left._{1}\right)$, note that, again because of coupling symmetry, $\mathbf{T}^{\mathrm{PK}}$ relates also $\left(A_{7}^{1}, A_{7}^{2}\right) \rightarrow\left(A_{5}^{2}, A_{5}^{1}\right)$, $\mathrm{T}^{\mathrm{KP}}$ does the same with $\left(A_{3}^{2}, A_{3}^{1}\right) \rightarrow\left(A_{1}^{1}, A_{1}^{2}\right)$, and $\mathrm{T}^{\mathbf{b}}$ connects also $\left(A_{5}^{2}, A_{4}^{2}, A_{5}^{1}\right) \rightarrow\left(A_{3}^{2}, A_{4}^{1}, A_{3}^{1}\right)$. 


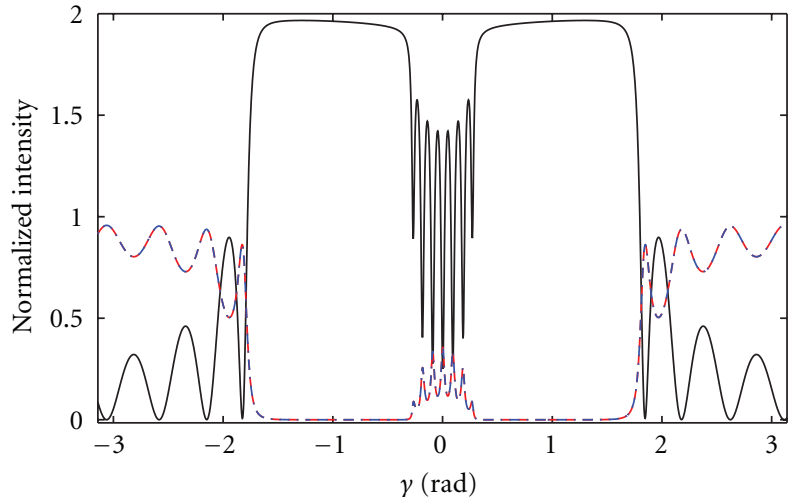

(a)

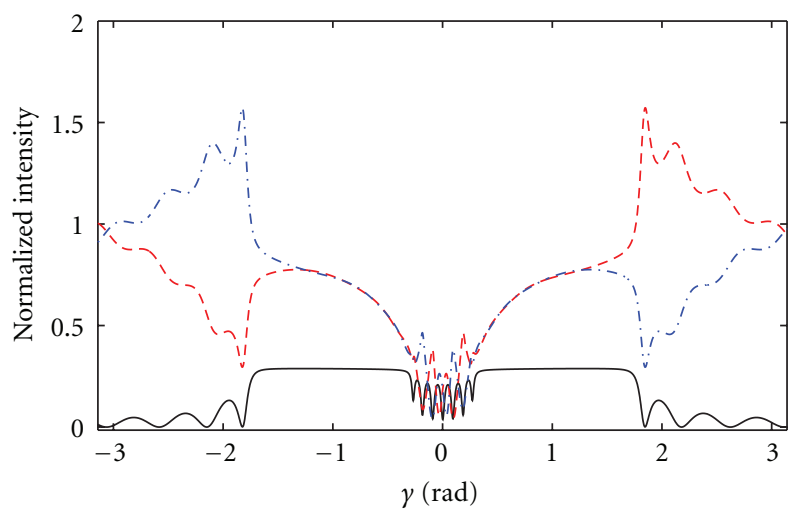

(c)

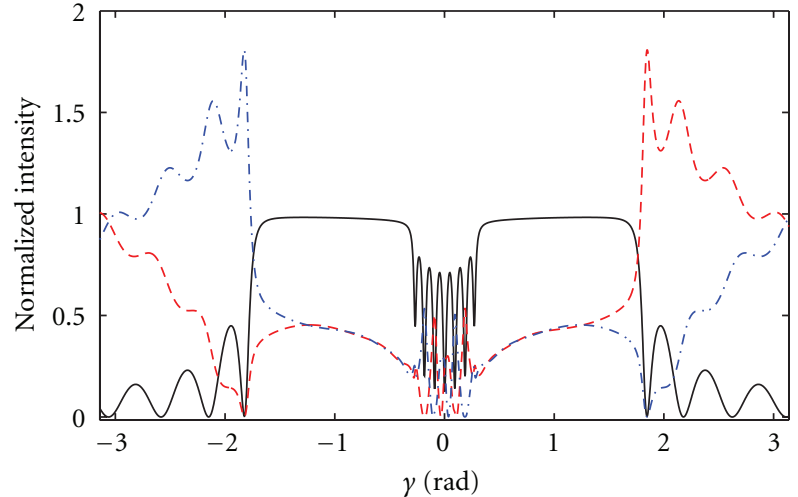

(b)

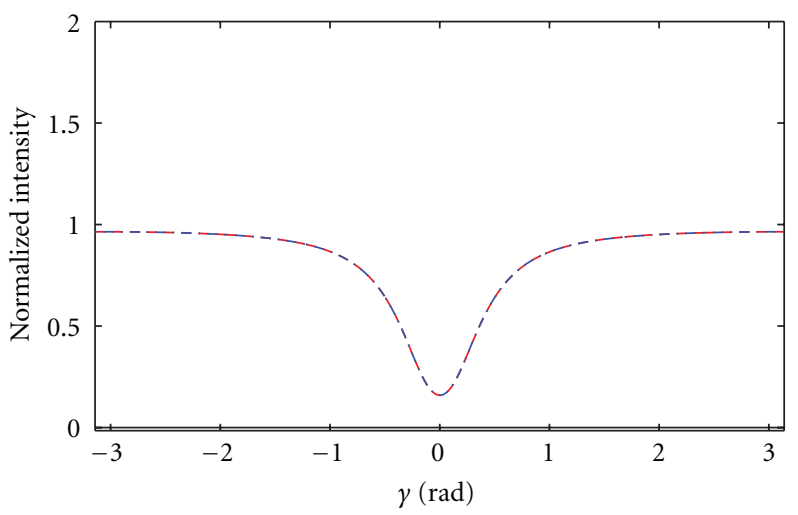

(d)

FIGURE 19: Spectral response of the $2 \times 8$ SCISSOR switch of Figure 16 with inputs, $A_{\operatorname{In}_{1}}=\left(1, e^{i(\pi / 2)}, e^{i(3 / 4) \pi}, e^{i \pi}\right)((\mathrm{a})$, (b), (c)), (d) resp.), $A_{\mathrm{Ad}}=0, A_{\mathrm{In}_{2}}=1$ (Lines as in Figure 12.)

Now, we can obtain the overall device response of Figure 11 by separating the contributions to the two Through and the Drop ports as the sum of three different terms corresponding to the three following device states.

State (I). Input signal in $\mathrm{In}_{1}$ only is injected and propagated from the top to the bottom of the device

$$
\left(\begin{array}{c}
A_{\mathrm{In}_{1}}=\left|A_{\mathrm{In}_{1}}\right| e^{i \phi_{1}} \\
A_{\mathrm{Ad}}=0 \\
A_{\mathrm{In}_{2}}=0
\end{array}\right) \longmapsto\left(\begin{array}{c}
A_{\mathrm{Th}_{1}^{(\mathrm{I})}} \\
A_{D^{(\mathrm{I})}} \\
A_{\mathrm{Th}_{2}^{(\mathrm{I})}}
\end{array}\right) .
$$
left):

Schematically, this goes as follows (from the right to the

$$
\begin{aligned}
& \longleftarrow\left(\begin{array}{c}
A_{3}^{2} \\
A_{3}^{1}
\end{array}\right)=\mathrm{T}^{\mathrm{PK}}\left(\begin{array}{c}
A_{\mathrm{In}_{1}} \\
A_{\mathrm{Th}_{1}^{(\mathrm{II}}}
\end{array}\right) \\
& \longleftarrow\left(\begin{array}{c}
A_{5}^{2} \\
A_{D^{(\mathrm{I})}} \\
A_{5}^{1}
\end{array}\right)=\mathrm{T}^{\mathbf{b}}\left(\begin{array}{c}
A_{3}^{2} \\
0 \\
A_{3}^{1}
\end{array}\right) \longleftarrow \\
& \left(\begin{array}{c}
0 \\
A_{\mathrm{Th}_{2}^{(\mathrm{II}}}
\end{array}\right)=\mathrm{T}^{\mathrm{KP}}\left(\begin{array}{c}
A_{5}^{2} \\
A_{5}^{1}
\end{array}\right) \longleftarrow .
\end{aligned}
$$

This means that the first set of equations from the first transferral of (B.5)

$$
\begin{aligned}
& A_{3}^{2}=T_{11}^{\mathrm{PK}} A_{\mathrm{In}_{1}}+T_{12}^{\mathrm{PK}} A_{\mathrm{Th}_{1}^{(\mathrm{I})},} \\
& A_{3}^{1}=T_{21}^{\mathrm{PK}} A_{\mathrm{In}_{1}}+T_{22}^{\mathrm{PK}} A_{\mathrm{Th}_{1}^{(1)}},
\end{aligned}
$$

have to be inserted into (B.6) to obtain

$$
\begin{aligned}
A_{5}^{2}= & \left(T_{11}^{b} T_{11}^{\mathrm{PK}}+T_{13}^{b} T_{21}^{\mathrm{PK}}\right) A_{\mathrm{In}_{1}} \\
& +\left(T_{11}^{b} T_{12}^{\mathrm{PK}}+T_{13}^{b} T_{22}^{\mathrm{PK}}\right) A_{\mathrm{Th}_{1}^{(\mathrm{I})}} \\
A_{D^{(\mathrm{II})}=} & \left(T_{21}^{b} T_{11}^{\mathrm{PK}}+T_{23}^{b} T_{21}^{\mathrm{PK}}\right) A_{\mathrm{In}_{1}} \\
& +\left(T_{21}^{b} T_{12}^{\mathrm{PK}}+T_{23}^{b} T_{22}^{\mathrm{PK}}\right) A_{\mathrm{Th}_{1}^{(\mathrm{I})}} \\
A_{5}^{1}= & \left(T_{31}^{b} T_{11}^{\mathrm{PK}}+T_{33}^{b} T_{21}^{\mathrm{PK}}\right) A_{\mathrm{In}_{1}} \\
& +\left(T_{31}^{b} T_{12}^{\mathrm{PK}}+T_{33}^{b} T_{22}^{\mathrm{PK}}\right) A_{\mathrm{Th}_{1}^{(1)} .}
\end{aligned}
$$

Proceeding further with the next transfer, we have from (B.7) that

$$
\begin{gathered}
0=T_{11}^{\mathrm{KP}} A_{5}^{2}+T_{12}^{\mathrm{KP}} A_{5}^{1}, \\
A_{\mathrm{Th}_{2}{ }^{(\mathrm{I})}}=T_{21}^{\mathrm{KP}} A_{5}^{2}+T_{22}^{\mathrm{KP}} A_{5}^{1},
\end{gathered}
$$




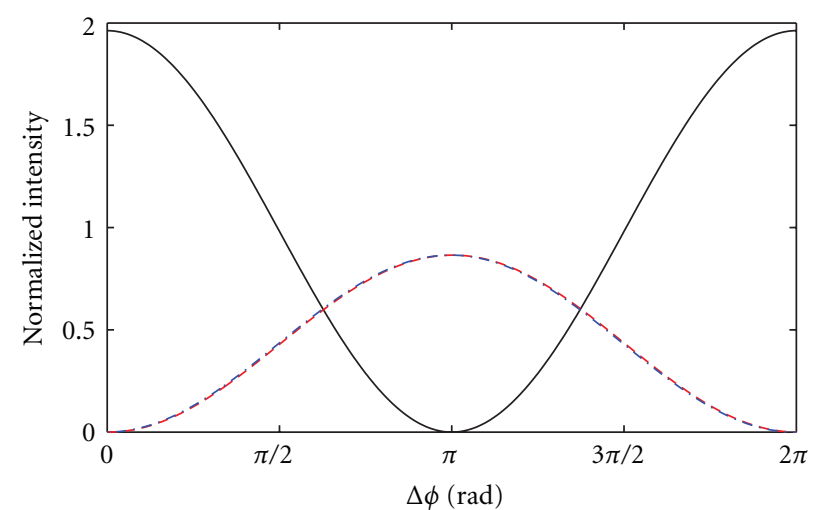

(a)

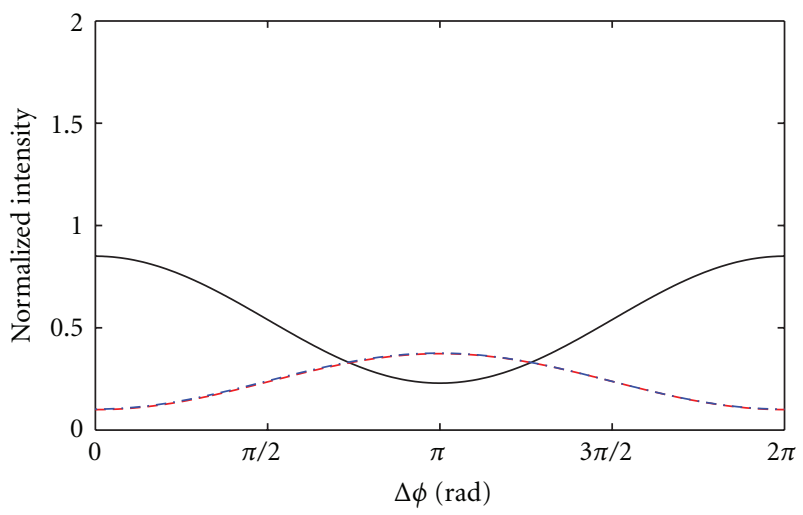

(c)

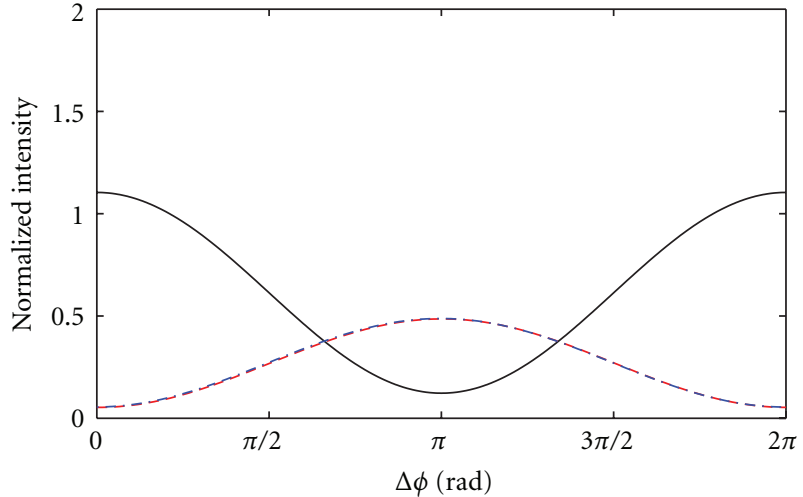

(b)

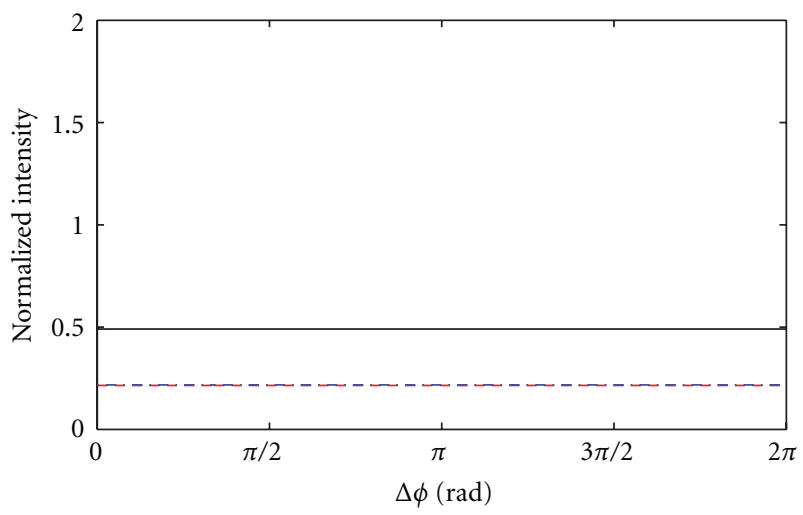

(d)

Figure 20: Phase response of the $2 \times 8$ SCISSOR switch of Figure 16 with input imbalances $\left|A_{\text {In }_{1}}\right|^{2}=(1,0.25,0.1,0)((a),(b),(c)$, (d), resp.), $A_{\mathrm{Ad}}=0, A_{\mathrm{In}_{2}}=1$, at phase round-trip shift $\gamma=1$ (Lines as in Figure 12.)

which, through (B.9), (B.10), and (B.11), leads to

$$
\begin{aligned}
& A_{\mathrm{Th}_{1}^{(\mathrm{I})}}=T_{11} A_{\mathrm{In}_{1}}, \\
& A_{D^{(\mathrm{I})}}=T_{21} A_{\mathrm{In}_{1}}, \\
& A_{\mathrm{Th}_{2}^{(\mathrm{I})}}=T_{31} A_{\mathrm{In}_{1}},
\end{aligned}
$$

with

$$
\begin{gathered}
T_{11}=-\frac{T_{11}^{\mathrm{KP}} A+T_{12}^{\mathrm{KP}} B}{T_{11}^{\mathrm{KP}} C+T_{12}^{\mathrm{KP}} D}, \\
T_{21}=E+F T_{11}, \\
T_{31}=G+H T_{11}, \\
A=T_{11}^{b} T_{11}^{\mathrm{PK}}+T_{13}^{b} T_{21}^{\mathrm{PK}}, \\
B=T_{31}^{b} T_{11}^{\mathrm{PK}}+T_{33}^{b} T_{21}^{\mathrm{PK}}, \\
C=T_{11}^{b} T_{12}^{\mathrm{PK}}+T_{13}^{b} T_{22}^{\mathrm{PK}}, \\
D=T_{31}^{b} T_{12}^{\mathrm{PK}}+T_{33}^{b} T_{22}^{\mathrm{PK}}, \\
E=T_{21}^{b} T_{11}^{\mathrm{PK}}+T_{23}^{b} T_{21}^{\mathrm{PK}}, \\
F=T_{21}^{b} T_{12}^{\mathrm{PK}}+T_{23}^{b} T_{22}^{\mathrm{PK}}, \\
G=T_{21}^{\mathrm{KP}} A+T_{22}^{\mathrm{KP}} B, \\
H=T_{21}^{\mathrm{KP}} C+T_{22}^{\mathrm{KP}} D .
\end{gathered}
$$

State (II). Input signal in $\mathrm{In}_{2}$ only is injected and propagated from the bottom to the top of the device.

$$
\left(\begin{array}{c}
A_{\mathrm{In}_{1}}=0 \\
A_{\mathrm{Ad}}=0 \\
A_{\mathrm{In}_{2}}=\left|A_{\mathrm{In}_{2}}\right| e^{i \phi_{2}}
\end{array}\right) \longmapsto\left(\begin{array}{c}
A_{\mathrm{Th}_{1}^{(\mathrm{III})}} \\
A_{D^{(\mathrm{III})}} \\
A_{\mathrm{Th}_{2}^{(\mathrm{III})}}
\end{array}\right) .
$$

One proceeds exactly as for State (I), but in the opposite direction. The only difference in the final result is that in (B.13) the ports $\left(\operatorname{In}_{1}, \operatorname{Through}_{1}^{(\mathrm{I})}\right.$, Through $\left.{ }_{2}^{(\mathrm{I})}\right)$ have to be exchanged with $\left(\operatorname{In}_{2}, \operatorname{Through}_{2}^{\text {(III) }}\right.$, Through $\left.{ }_{1}^{(\mathrm{III})}\right)$ :

$$
\begin{gathered}
A_{\mathrm{Th}_{1}^{(\mathrm{III})}}=T_{13} A_{\mathrm{In}_{2}}, \\
A_{\mathrm{Drop}^{(\text {III) }}}=T_{23} A_{\mathrm{In}_{2}}, \\
A_{\mathrm{Th}_{2}^{(\text {III) }}}=T_{33} A_{\mathrm{In}_{2}},
\end{gathered}
$$

with $T_{13}=T_{31}, T_{23}=T_{21}$, and $T_{33}=T_{11}$.

State (III). Add port signal only is injected and propagated from the central Add waveguide towards the Drop and upper and lower Through ports.

In the transfer matrix of (35), only one coefficient is missing, namely, $T_{22}$. This is fixed by the other matrix elements and power conservation criteria. Expressing explicitly 


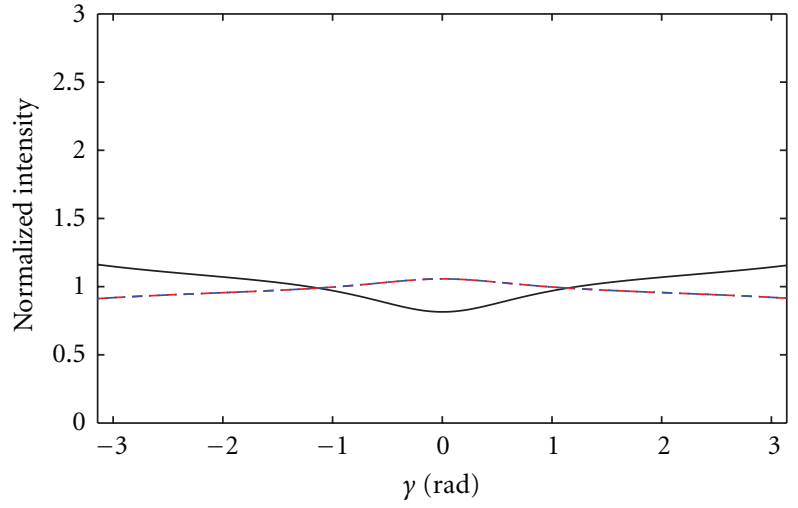

(a)

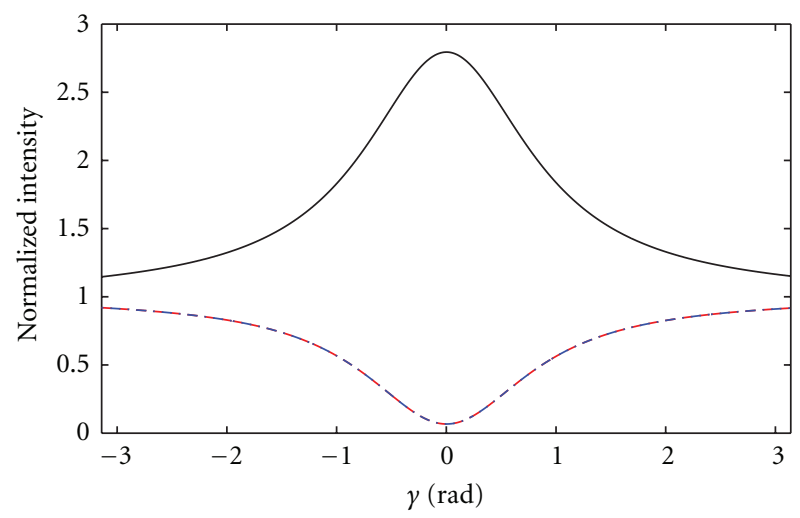

(c)

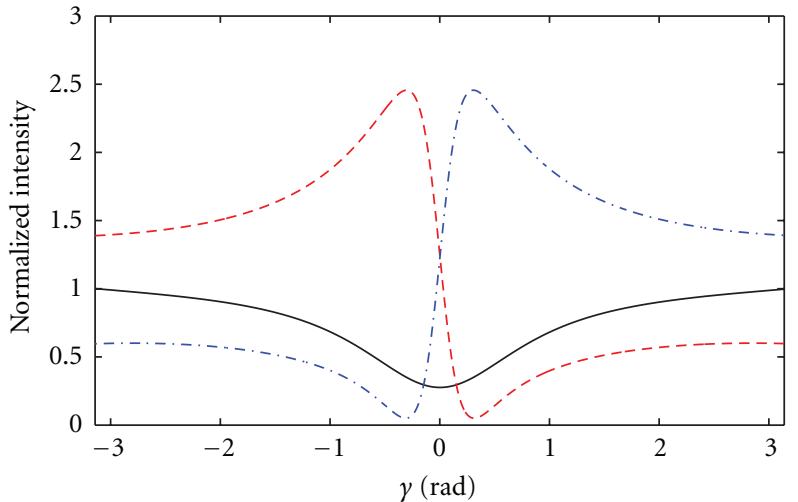

(b)

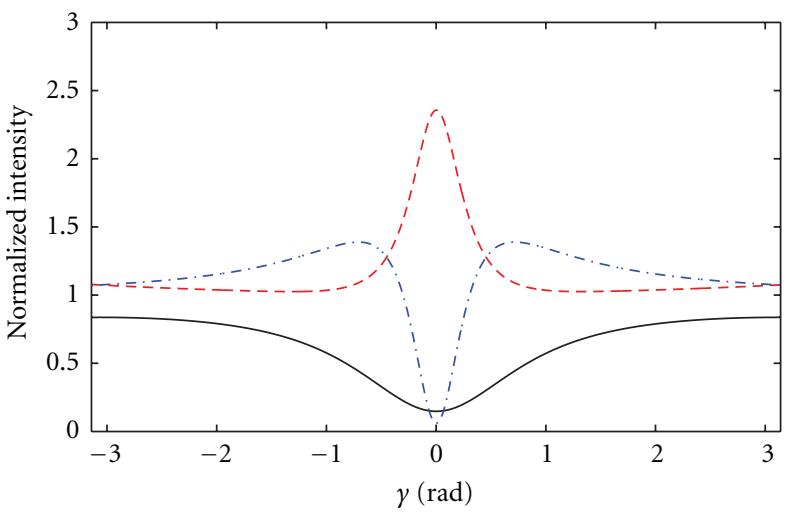

(d)

FIGURE 21: Spectral response of the $2 \times 1$ SCISSOR switch of Figure 16 with inputs $A_{\text {In } 1}=e^{i \phi_{1}}, A_{\text {Ad }}=e^{i \phi_{A_{d d}}}, A_{\text {In }_{2}}=e^{i \phi_{2}}$, and $\left(\phi_{1}, \phi_{\mathrm{Add}_{2}}, \phi_{2}\right)$ $=(0,0,0) ;\left(\phi_{1}, \phi_{\text {Add }}, \phi_{2}\right)=(0, \pi / 4, \pi / 2) ;\left(\phi_{1}, \phi_{\text {Add }}, \phi_{2}\right)=(0, \pi, 0) ;\left(\phi_{1}, \phi_{\text {Add }}, \phi_{2}\right)=(0, \pi, \pi)$ for figures (a), (b), (c), (d), respectively (Lines as in Figure 12.)

with the matrix elements of $\mathbf{T}$ the power balance between the input and output intensities of the waves, it must hold:

$$
\begin{aligned}
&\left|A_{\mathrm{In}_{1}}\right|^{2}+\left|A_{\mathrm{In}_{2}}\right|^{2}+\left|A_{\mathrm{Ad}}\right|^{2} \\
&=\left|A_{\mathrm{Th}_{1}}\right|^{2}+\left|A_{D}\right|^{2}+\left|A_{\mathrm{Th}_{2}}\right|^{2}+\text { Loss } \\
&=\left(\left|T_{11}\right|^{2}+\left|T_{21}\right|^{2}+\left|T_{31}\right|^{2}\right)\left(\left|A_{\mathrm{In}_{1}}\right|^{2}+\left|A_{\mathrm{In}_{2}}\right|^{2}\right) \\
&+\left(2\left|T_{21}\right|^{2}+\left|T_{22}\right|^{2}\right)\left|A_{\mathrm{Ad}}\right|^{2} \\
&+\left(T_{11} T_{31}^{*}+\left|T_{21}\right|^{2}+T_{31} T_{11}^{*}\right)\left(A_{\mathrm{In}_{1}} A_{\operatorname{In}_{2}}^{*}+A_{\mathrm{In}_{1}}^{*} A_{\mathrm{In}_{2}}\right) \\
&+\left(T_{11} T_{21}^{*}+T_{21} T_{22}^{*}+T_{31} T_{21}^{*}\right)\left(A_{\mathrm{In}_{1}}+A_{\mathrm{In}_{1}}\right) A_{\mathrm{Ad}}^{*} \\
&+\left(T_{11}^{*} T_{21}+T_{21}^{*} T_{22}+T_{31}^{*} T_{21}\right)\left(A_{\mathrm{In}_{1}}^{*}+A_{\mathrm{In}_{2}}\right) A_{\mathrm{Ad}}+\text { Loss, }
\end{aligned}
$$

with the last term indicating the losses of the structure. This implies the following conditions:

$$
\begin{gathered}
\left|T_{11}\right|^{2}+\left|T_{21}\right|^{2}+\left|T_{31}\right|^{2}=1, \\
2\left|T_{21}\right|^{2}+\left|T_{22}\right|^{2}=1,
\end{gathered}
$$

$$
\begin{aligned}
& T_{11} T_{31}^{*}+\left|T_{21}\right|^{2}+T_{31} T_{11}^{*}=0, \\
& T_{11}^{*} T_{21}+T_{21}^{*} T_{22}+T_{31}^{*} T_{21}=0 .
\end{aligned}
$$

Conditions (B.18) and (B.20) are already satisfied by the coefficients found in the two previous cases (very cumbersome and long calculations). From (B.21), we finally obtain the last coefficient for matrix (35):

$$
T_{22}=-\frac{T_{21}}{T_{21}^{*}}\left(T_{11}^{*}+T_{31}^{*}\right)
$$

which satisfies also (B.19) through (B.20).

\section{Acknowledgment}

The authors acknowledge support of the EU through the FP7 ICT-(216405) Project Wavelength Division Multiplexed Photonic Layer on CMOS.

\section{References}

[1] T. S. El-Bawab, Optical Switching, Springer, 2006. 
[2] A. Yariv, Y. Xu, R. K. Lee, and A. Scherer, "Coupled-resonator optical waveguide: a proposal and analysis," Optics Letters, vol. 24, no. 11, pp. 711-713, 1999.

[3] J. E. Heebner, R. W. Boyd, and Q. H. Park, "SCISSOR solitons and other novel propagation effects in microresonatormodified waveguides," Journal of the Optical Society of America B, vol. 19, no. 4, pp. 722-731, 2002.

[4] J. E. Heebner, P. Chak, S. Pereira, J. E. Sipe, and R. W. Boyd, "Distributed and localized feedback in microresonator sequences for linear and nonlinear optics," Journal of the Optical Society of America B, vol. 21, no. 10, pp. 1818-1832, 2004.

[5] J. Capmany, P. Muñoz, J. D. Domenech, and M. A. Muriel, "Apodized coupled resonator waveguides," Optics Express, vol. 15, no. 16, pp. 10196-10206, 2007.

[6] S. Y. Cho and R. Soref, "Apodized SCISSORs for filtering and switching," Optics Express, vol. 16, no. 23, pp. 19078-19090, 2008.

[7] D. U. Smith, H. Chang, K. A. Fuller, A. T. Rosenberger, and R. W. Boyd, "Coupled-resonator-induced transparency," Physical Review A, vol. 69, no. 6, Article ID 063804, 6 pages, 2004.

[8] Q. Xu, S. Sandhu, M. L. Povinelli, J. Shakya, S. Fan, and M. Lipson, "Experimental realization of an on-chip all-optical analogue to electromagnetically induced transparency," Physical Review Letters, vol. 96, no. 12, Article ID 123901, 4 pages, 2006.

[9] Y. F. Xiao, X. B. Zou, W. Jiang, Y. L. Chen, and G. C. Guo, "Analog to multiple electromagnetically induced transparency in all-optical drop-filter systems," Physical Review A, vol. 75, no. 6, Article ID 063833, 4 pages, 2007.

[10] X. Yang, M. Yu, D. L. Kwong, and C. W. Wong, "All-optical analog to electromagnetically induced transparency in multiple coupled photonic crystal cavities," Physical Review Letters, vol. 102, no. 17, Article ID 173902, 4 pages, 2009.

[11] M. Mancinelli, R. Guider, M. Masi et al., "Optical characterization of a SCISSOR device," Optics Express, vol. 19, no. 14, pp. 13664-13674, 2011.

[12] M. Masi, M. Mancinelli, A. Battarelli et al., "A silicon photonic interferometric router device based on SCISSOR concept," Journal of Lightwave Technology, vol. 29, no. 18, pp. 2747-2753, 2011.

[13] "Technology focus on Lithography," Nature Photonics, vol. 4, no. $1,2010$.

[14] G. T. Reed, G. Mashanovich, F. Y. Gardes, and D. J. Thomson, "Silicon optical modulators," Nature Photonics, vol. 4, article $660,2010$.

[15] K. Okamoto, Fundamentals of Optical Waveguides, Academic Press, 2nd edition, 2006.

[16] Y. M. Landobasa, S. Darmawan, and M. K. Chin, "Matrix analysis of 2-d microresonator lattice optical filters," IEEE Journal of Quantum Electronics, vol. 41, no. 11, pp. 1410-1418, 2005.

[17] J. K. S. Poon, J. Scheuer, S. Mookherjea, G. T. Paloczi, Y. Huang, and A. Yariv, "Matrix analysis of microring coupledresonator optical waveguides," Optics Express, vol. 12, no. 1, pp. 90-103, 2004.

[18] C.-S. Ma, X. Yan, and X.-Y. Wang, "Matrix analysis of 2D microring resonator arrays," Journal of Modern Optics, vol. 55, no. 1, pp. 143-154, 2008.

[19] J. K. S. Poon, P. Chak, J. M. Choi, and A. Yariv, "Slowing light with Fabry-Perot resonator arrays," Journal of the Optical Society of America B, vol. 24, no. 11, pp. 2763-2769, 2007.

[20] S. Y. Cho and R. Soref, "Interferometric microring-resonant $2 \times 2$ optical switches," Optics Express, vol. 16, no. 17, pp. 13304-13314, 2008.
[21] F. Xia, L. Sekaric, M. O’Boyle, and Y. Vlasov, "Coupled resonator optical waveguides based on silicon-on-insulator photonic wires," Applied Physics Letters, vol. 89, no. 4, Article ID 041122, 3 pages, 2006.

[22] M. Masi, R. Orobtchouk, G. Fan, J. M. Fedeli, and L. Pavesi, "Towards a realistic modelling of ultra-compact racetrack resonators," Journal of Lightwave Technology, vol. 28, no. 22, pp. 3233-3242, 2010.

[23] U. Fano, "Sullo spettro di assorbimento dei gas nobili presso il limite dello spettro d'arco," Nuovo Cimento, vol. 12, no. 3, pp. 154-161, 1935.

[24] U. Fano, "Effects of configuration interaction on intensities and phase shifts," Physical Review, vol. 124, no. 6, pp. 18661878, 1961. 

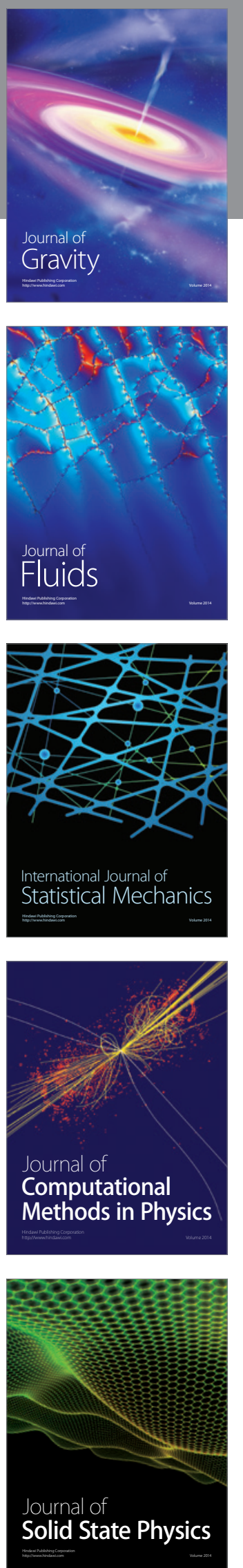

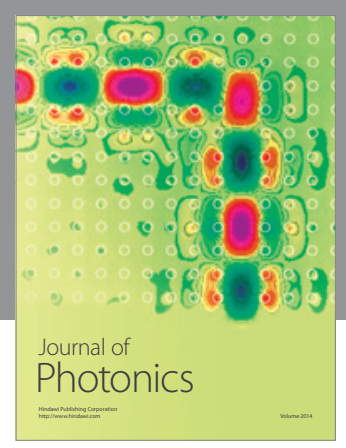

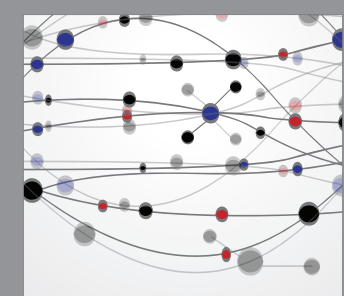

The Scientific World Journal
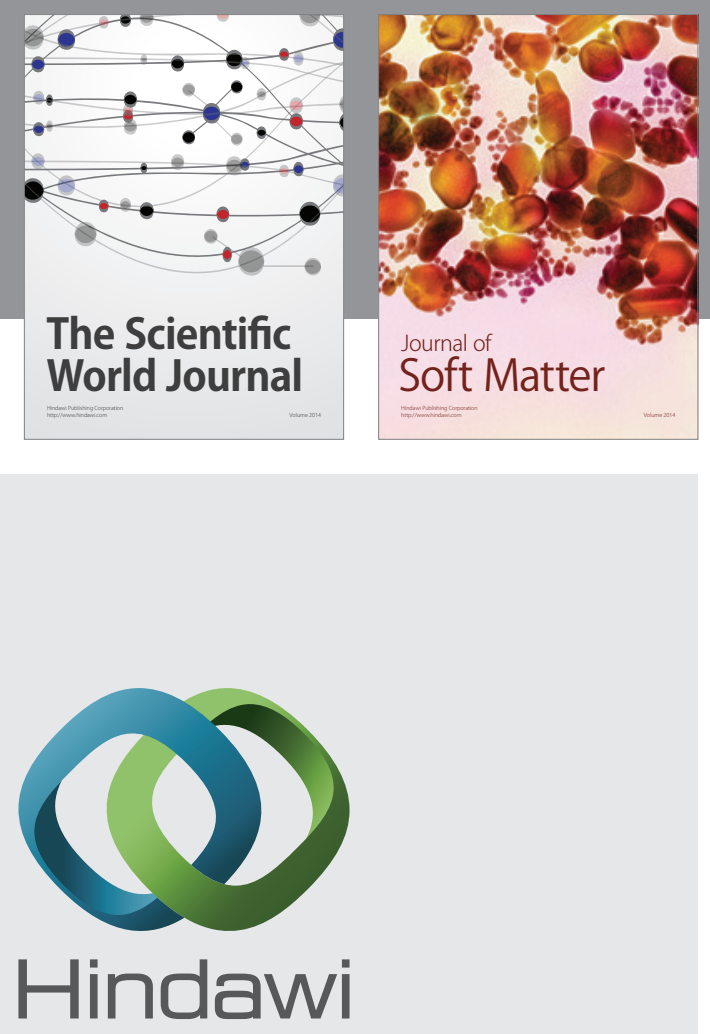

Submit your manuscripts at

http://www.hindawi.com
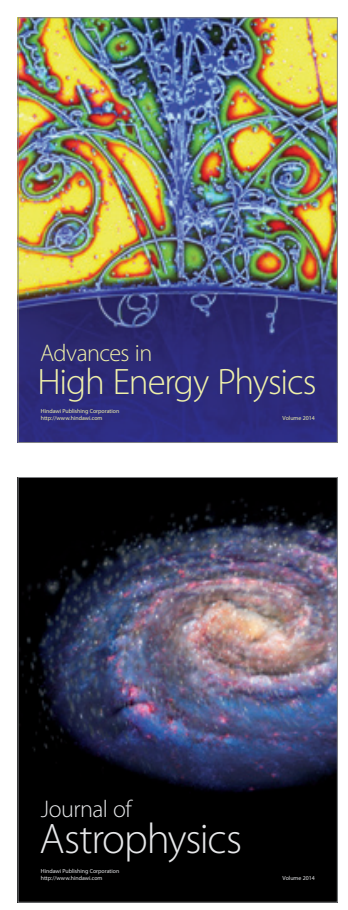
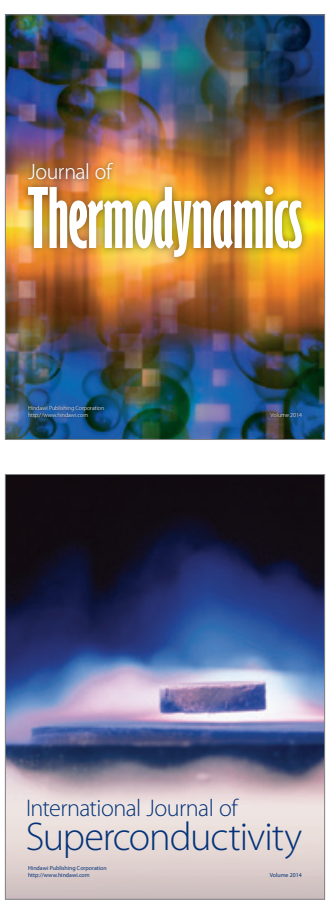
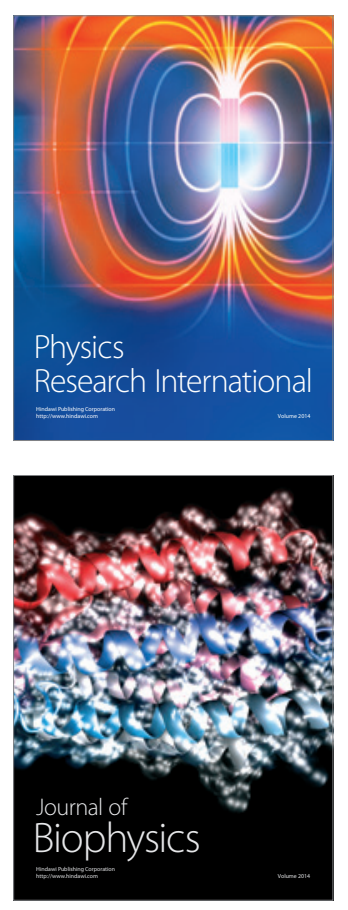
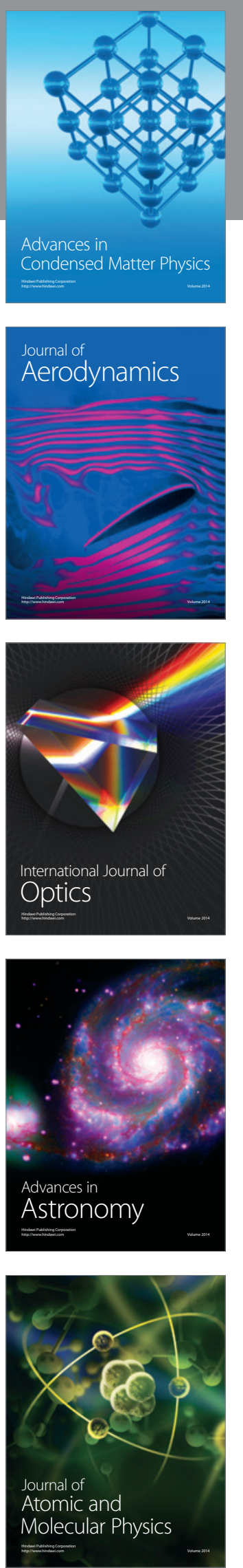